\title{
An alternative approach to the galactic dark matter problem
}

\author{
Ulises Nucamendi* \\ Centre for Theoretical Physics \\ University of Sussex \\ Brighton BN1 9QJ, Great Britain \\ Marcelo Salgado ${ }^{\dagger}$ and Daniel Sudarsky ${ }^{\ddagger}$ \\ Instituto de Ciencias Nucleares \\ Universidad Nacional Autónoma de México \\ Apdo. Postal 70-543 México 04510 D.F, México.
}

\begin{abstract}
We discuss scenarios in which the galactic dark matter in spiral galaxies is described by a long range coherent field which settles in a stationary configuration that might account for the features of the galactic rotation curves. The simplest possibility is to consider scalar fields, so we discuss in particular, two mechanisms that would account for the settlement of the scalar field in a non-trivial configuration in the absence of a direct coupling of the field with ordinary matter: topological defects, and spontaneous scalarization.
\end{abstract}

PACS number(s): 11.27.+d, 04.40.-b, 98.62.Gq

\footnotetext{
*e-mail: ung@star.cpes.susx.ac.uk

†e-mail: marcelo@nuclecu.unam.mx

‡e-mail: sudarsky@nuclecu.unam.mx
} 


\section{INTRODUCTION}

It has been known for a long time that the motion of the stars and gases around the center of most galaxies can not be explained in terms of the luminous matter content of the galaxies, at least not within the context of Newtonian gravity (see [1] for a review). The standard view is that there is in almost every galaxy a large component of non-luminous matter (the gravitational dark matter) that forms an halo around the galaxy and that provides the additional gravitational attraction needed to explain the "rotation curves" in terms of standard gravitational theory. There are several proposals for this dark component, ranging from new exotic particles such as those predicted by supersymmetry [2 4], to other less exotic candidates such as massive neutrinos, all collectively known as WIMPs (weakly interacting massive particles) (see [2.5] for a review), to the relative mundane idea of dark but ordinary bodies such as Jupiter-like objects collectively known as MACHOs (Massive Compact Halo Objects) [6]. Searches for these types of objects have been made [7], and although they report some findings, there doesn't seem to be enough of these objects to account for the galactic dynamics. Moreover there are severe bounds on the amount of baryonic matter in the universe arising from big bang nucleosynthesis and for some values of the Hubble constant those bounds also imply that some of the galactic dark matter ought to be exotic [8 10]. Independently of this and despite their popularity, these type of models suffer from various problems and require surprising coincidences (see for example [1]]).

Another type of proposal, which is in some sense more radical, is based on the idea that the gravitational theory would have to be modified when dealing with the scales associated with the motion of stars in galaxies [12,13, in particular the idea is embodied by the proposal of Milgrom [14], that the laws of motion are modified when the accelerations involved are extremely small. Unfortunately this scenario has not, as yet, been converted into a fully relativistically invariant theory. Another type of model that has been exposed is to replace general relativity by a higher order in curvature theory, which in some particular cases appears to be obtaining encouraging results 15. The problem with this approach is that these types of theories have in general problems of principle like for example the lack of a well posed initial value formulation. Nevertheless such relatively radical proposals are still attractive, due in part to an intrinsic problems of the more conservative approaches in explaining the generality and universality of the phenomena, namely the fact that the amount of luminous matter seems to be such a good indicator of the amount of the dark matter component [16] and the fact that the dark component happens always to distribute itself in such a way that the resulting rotation curves (hereafter referred to as RC) are almost flat away from the galactic centers [17]. 
Thus, in contrast with the former scenarios which would need to assume not only the existence of the dark matter but also give some evolutionary scenarios that result in the aforementioned universality in its distribution, the modified gravity scenario would naturally account for such correlations without the need for additional assumptions. On the other hand the former scenarios do not present any problem in lending themselves to an acceptable theoretical formulation, compatible with present theories of particle physics and general relativity.

The object of this article is to discuss a third type of scenario which has some of the advantages of each scheme. The idea is to take dark matter to be described not by a bunch of particles whose distribution needs to be explained but by a coherent field which would settle in a universal stationary configuration that would account for the generic features of the RC. The simplest possibility is provided by scalar fields, which would of course have to be very long ranged (i.e. masses smaller than $\frac{1}{R_{G}}$ where $R_{G}$ is the radius of the largest galaxy with flat $\mathrm{RC}$ ). The basic problem is that there are very severe experimental bounds for the direct coupling of such a field with ordinary matter [18], and in the absence of such coupling the field will in general settle globally in the minimum of the potential leading to a homogeneous configuration that will not produce the desired effects. On the other hand, one could hope that, given the likelihood of existence of large black holes at the center of most galaxies, they would account for the nontrivial configuration of the scalar fields. Unfortunately these kind of situation is largely forbidden by the "Black Hole No hair theorems" for scalar fields [19 21]. These limitations severely reduce the types of models one can consider, in particular, there are, known to these authors, only three mechanisms that would account for the settlement of the scalar field in a non-trivial configuration in the absence of a direct coupling of the field with ordinary matter (or some other exotic matter which we will not consider because of the incremental number of hypothesis it involves): a) boson-star like clumps, b) spontaneous scalarization, and c) topological defects. Other models that lack these features have been considered, for example in [22]. However, such models face two problems: first, they give rise to spherical configurations where the scalar field in consideration is singular "at the center", and second, the resulting scalar field potential needed to account for the flat $\mathrm{RC}$ depends explicitly on the value of the "tangential velocity" of stars at the flat region. That is to say, such a potential have to be adjusted differently for different galaxies. Needless to say that both problems clearly make those schemes unsuitable as models for the problem at hand.

Concerning the case "a)" mentioned above, it has been analyzed in [23]. Their analysis focuses on cosmological and evolutionary considerations as well as the issues related to the conditions under which the assumption of long range coherency of the scalar field is justified, rather than the universal features of the galactic rotation curves. We will deal here with the other two cases "b)" and "c)". 
The scenario "b)", namely the spontaneous scalarization (see Sec.V) 24, is in some sense simpler because it involves a single scalar field in contrast of the various fields needed in the simplest versions of topological defects (e.g., global monopoles). Here the mechanism that allows for the nontrivial stationary configuration of the scalar field is connected to a non-minimal coupling of the scalar field to the curvature. This results in the effective gravitational coupling becoming dependent on the scalar field. The point is that such a coupling allows for the reduction of the total energy of the configuration (in comparison with the corresponding configuration with the same baryon number and no scalar field) for which the scalar field deviates from the trivial configuration by taking values that reduce the gravitational coupling in the regions of high matter density [25]. Thus the model must incorporate from the onset the non-minimal coupling that seems to be needed to account at the same time for the correlations in the dark-luminous matter components (see [34] and the discussion of the third scenario below). The disadvantage of this model, which is in fact shared by the first model (i.e., boson stars) [26], is precisely the lack of resilience against black holes whose existence in most galaxies, if confirmed, would seem to preclude, through the no-hair theorems 19 21], the models based on this mechanism.

The scenario "c)" is exemplified by the model of global monopoles [32] which have the notable feature of naturally leading to a $1 / r^{2}$ energy density behavior which would naively account for the flat rotation curves and which upon taking the symmetry braking scale to be the GUT scale would result in the correct order of magnitude for the galactic dark matter. Unfortunately, upon further examination of the simplest model severe problems arise, in particular the monopole configuration turns out to be repulsive [33], and moreover the configuration would be too universal in the sense that it would be independent of the size of the galaxy thus defeating the hope for the correlation of dark to luminous matter over a range of galactic sizes. There is nevertheless hope to overcome these problems by the consideration of slightly more complicated models [34]. In that work the simple monopole model was supplemented by the introduction of a non-minimal coupling between the scalar fields and curvature (see [34] and Sec.VI). This resulted in the restoration of gravitational attraction leading to regions of relatively flat rotation curves and to the possibilities of the dark-luminous matter correlations arising from the fact that in these models the scalar potential $V\left(\Phi^{a} \Phi_{a}\right)$ (where $\Phi^{a}$ stands for a triplet of scalar fields that characterize the global monopole) is replaced with the effective scalar potential $V\left(\Phi^{a} \Phi_{a}\right)+F\left(\Phi^{a} \Phi_{a}, R\right)$ (here $R$ stands for the scalar curvature of the space-time metric) whose minima depend on the amount of matter present trough the effect of the latter on $R$. The global monopole model has the additional advantage of resilience against the formation of black holes in the galactic centers, since their topological charge makes them immune to the devastating limitations imposed by the no hair 
theorems.

Despite the promising features of the model "c)", our intention in the present work is to take a step backwards and look at the problem from a more general point of view before embarking in the methodical study of a particular type of model.

The article is organized as follows: in Section II, we analyze the generic form of the rotation curves of galaxies in a general relativistic context. In section III, we comment on the Newtonian approximation and on the embedding of the galaxy in the large scale space-time. In Section IV, we discuss the additional information that can be obtained about the metric from other considerations, specifically the deflection of light by the galaxy. Section V reviews the spontaneous scalarization scenarios. In Section VI, we review the non-minimally coupled global monopole model and discuss its shortcomings. Finally, in Section VII we offer a discussion and analyze the directions for further developments.

\section{ROTATION CURVES OF GALAXIES AND FREQUENCY SHIFTS}

The rotation curves (RC) provides the most direct method of analyzing the gravitational field inside a spiral galaxy. $\mathrm{RC}$ have been determined for a great amount of spiral galaxies 16, 17]. They are obtained by measuring the frequency shifts of light emitted from stars and from the $21 \mathrm{~cm}$ radiation from neutral gas clouds.

In fact, since (apart from the central regions) the "tangential velocity" of rotation $v$ remains approximately constant up to distances far beyond the luminous radius of these galaxies, a naive Newtonian analysis leads to the conclusion that the energy density decreases with the distance as $r^{-2}$ and therefore that the mass of galaxies increases as $m(r) \approx r$. On the other hand, one could naturally question whether these large mass ought not to result into an important gravitational redshift. We will carry our analysis in a general relativistic setting and will see in the following sections that with standard assumptions about the matter content of the galaxy, the behavior of the $\mathrm{RC}$ indicates that the spacetime is not in general described by the standard form $d s^{2}=-(1+2 \Phi) d t^{2}+(1+2 \Phi)^{-1} d r^{2}+r^{2} d \Omega$ as can be initially thought (see for example [23]).

In order to analyze the problem we will focus directly on what it is observed because only then will we be able to discuss models that do not let themselves

to a Newtonian based inferences. This is an important point since the lack of understanding of it leads to erroneous conclusions [35].

The observations of stars and gas in spiral galaxies show a shift $z_{\text {tot }}$ in their 
intrinsic spectra which includes the contributions of: 1) the cosmological expansion (recession of galaxies), 2) the peculiar motion of the galaxy, 3) the thermal motion of atoms within the stars and gas, 4) the gravitational field within the galaxy and stars, and finally 5) the motion of the stars around the galactic center.

When the "contaminating" effects from 1)-3) are subtracted from the data, usually astronomers report the resulting $z$ in terms of a velocity field $v$. Nevertheless it is instructive to make the analysis in terms of the quantities that are most directly observable: the $z$ 's. We perform this in order to keep track of the effect of the underlying assumptions, and to enable us to carry the analysis when these are not longer valid, as will be the case in some models we will consider.

The starting point is to assume that stars behave like test particles which follow geodesics of a static and spherically symmetric space-time associated with sources that we do not specify for the moment. The most general line element of the space-time in these circumstances takes the form:

$$
d s^{2}=-N^{2}(r) d t^{2}+A^{2}(r) d r^{2}+r^{2} d \theta^{2}+r^{2} \sin ^{2} \theta d \varphi^{2} .
$$

Next we consider two observers $\mathcal{O}_{E}$ and $\mathcal{O}_{D}$ with four velocities $u_{E}^{\mu}, u_{D}^{\mu}$ respectively. Observer $\mathcal{O}_{E}$ corresponds to the light emitter (i.e., to the stars placed at a point $P_{E}$ of space-time), and $\mathcal{O}_{D}$ represents the detector at point $P_{D}$ located far from the emitter and that can be idealized to correspond to "spatial infinity".

Without loss of generality, we can assume that the stars move on the galactic plane $\theta=\pi / 2$, so that $u_{E}^{\mu}=(\dot{t}, \dot{r}, 0, \dot{\varphi})_{E}$, where the dot stands for derivation with respect to the proper time of the particle.

On the other hand, we suppose that the detector is static (i.e, $\mathcal{O}_{D}$ 's 4 -velocity is tangent to the static Killing field $\frac{\partial}{\partial t}$ ), and so with respect to the above coordinates its 4 -velocity is $u_{D}^{\mu}=(\dot{t}, 0,0,0)_{D}$.

As usual, the consideration of the norm of the four velocity $\left(u^{\mu} u_{\mu}=-1\right)$, gives,

$$
-1=-N^{2}(r)\left(\dot{t}^{2}\right)+A^{2}\left(\dot{r}^{2}\right)+r^{2}\left(\dot{\varphi}^{2}\right),
$$

The energy and the angular momentum per unit of mass at rest of the test particle are conserved quantities and can be written as

$$
E=-g_{\mu \nu} \varepsilon^{\mu} u^{\nu}=N^{2}(\dot{t}), \quad L=g_{\mu \nu} \psi^{\mu} u^{\nu}=r^{2}(\dot{\varphi}),
$$

where $\varepsilon^{\mu}, \psi^{\mu}$ denote the timelike and rotational killing fields of the metric (1) respectively. Introducing these constants of motion in the line element (2), we obtain 


$$
N^{2} A^{2}(\dot{r})^{2}+N^{2}\left[\frac{L^{2}}{r^{2}}+1\right]=E^{2},
$$

This equation shows that the radial motion of a geodesic is the same as that of a particle with position dependent mass and with energy $E^{2} / 2$ in ordinary non-relativistic mechanics moving in the effective potential

$$
V_{e f f}(r)=N^{2}(r)\left[\frac{L^{2}}{r^{2}}+1\right] .
$$

As we mentioned, the $\mathrm{RC}$ of spiral galaxies are inferred from the red and blue shifts of the emitted radiation by stars moving in "circular orbits" on both sides of the central region [17]. The light signal travels on null geodesics with tangent $k^{\mu}$. We may restrict $k^{\mu}$ to lie also in the "equatorial plane" $\theta=\pi / 2$, and evaluate the frequency shift for a light signal emitted from $\mathcal{O}_{E}$ in circular orbit and detected by $\mathcal{O}_{D}$. The conditions for circular orbits $\partial_{r} V_{\text {eff }}=0$ and $\dot{r}=0$ lead to

$$
\begin{aligned}
L^{2} & =\frac{r^{3} \partial_{r} N / N}{1-r \partial_{r} N / N}, \\
E^{2} & =\frac{N^{2}}{1-r \partial_{r} N / N}
\end{aligned}
$$

The frequency shift associated to the emission and detection is given by,

$$
z=1-\frac{\omega_{E}}{\omega_{D}}
$$

where

$$
\omega_{C}=-\left.k_{\mu} u_{C}^{\mu}\right|_{P_{C}}
$$

the index $C$ refers to emission or detection at the corresponding space-time point.

Two frequency shifts corresponding to a maximum and minimum values are associated with light propagation in the same and opposite direction of motion of the emitter respectively (i.e., $k^{r}=k^{\theta}=0$ ). Such shifts are the frequency shifts of a receding or approaching star respectively. Using the constancy along the geodesic of the product of the Killing field $\frac{\partial}{\partial t}$ with a geodesic tangent together with (9) and (8), and expressions (7) and (6), we find the two shifts to be,

$$
z_{ \pm}=1-\frac{N_{D}}{N(r)} \frac{\left(1 \mp\left[r \partial_{r} N(r) / N(r)\right]^{1 / 2}\right)}{\left[1-r \partial_{r} N(r) / N(r)\right]^{1 / 2}}
$$

where $N(r)$ represents the value of the metric potential at the radius of emission $r$, and $N_{D}$ the corresponding value of $N(r)$ at $r \rightarrow \infty$ where the detector 
is supposed to lie. For asymptotically flat space-times $N_{D} \rightarrow 1$. However, for space-times generated by global-monopoles $N_{D} \rightarrow(1-\alpha)^{1 / 2}$ (see the section $\mathrm{VI})$.

It is worth noting that in terms of the tetrads $e_{(0)}=N^{-1} \frac{\partial}{\partial t}, e_{(1)}=$ $A^{-1} \frac{\partial}{\partial r}, e_{(2)}=r^{-1} \frac{\partial}{\partial \theta}, e_{(3)}=(r \sin \theta)^{-1} \frac{\partial}{\partial \phi}$, the frequency shifts take the form,

$$
z_{ \pm}=1-\frac{N_{D}}{N}(1 \mp v) \Gamma
$$

where $v:=\left(\sum_{i=1,2,3}\left(u_{(i)} / u_{(0)}\right)^{2}\right)^{1 / 2}$ and $u_{(\mu)}$ stands for the components of the star's four velocity along the tetrad (i.e., the velocity measured by an Eulerian observer whose world line is tangent to the static Killing field) and $\Gamma=$ $\left(1-v^{2}\right)^{-1 / 2}$ is the usual Lorentz factor. Clearly, in the present case of circular orbits on the plane $\theta=\pi / 2$, it results that $v=u_{(3)} / u_{(0)} \equiv r \partial_{r} N(r) / N(r)$.

It is convenient to define the quantities: $z_{D}=\frac{1}{2}\left(z_{+}-z_{-}\right)$and $z_{A}=\frac{1}{2}\left(z_{+}+z_{-}\right)$ which are easily connected to the observations. From the expression (10) we obtain,

$$
\begin{gathered}
z_{D}(r)=\frac{N_{D}}{N(r)} \frac{\left[r \partial_{r} N(r) / N(r)\right]^{1 / 2}}{\left[1-r \partial_{r} N(r) / N(r)\right]^{1 / 2}} . \\
z_{A}(r)=1-\frac{N_{D}}{N(r)} \frac{1}{\left[1-r \partial_{r} N(r) / N(r)\right]^{1 / 2}} .
\end{gathered}
$$

We note, for example, that $\left(z_{A}-1\right)^{2}-z_{D}^{2}=\left(N(r) / N_{D}\right)^{-2}$, and thus we could in principle recover $N(r)$ directly from the observations. Then we can use this $N(r)$ to recalculate $z_{A}$ and $z_{D}$ from the above expressions and compare them with the measured values. This would be a test of the assumption that the dynamics is determined by the geodesics of a stationary metric, quite independently of the assumption of the dynamics of the geometry itself or for of the nature of the dark matter.

\section{GRAVITATIONAL FIELD IN THE DARK MATTER ZONE}

In this section we will use the form of the $\mathrm{RC}$ to obtain the spacetime metric and information on the matter content. The energy-momentum tensor must be diagonal and spherically symmetric, as dictated by the symmetries of the space-time (11), so we define:

$$
\rho \equiv-T_{t}^{t} \quad, \quad P_{r} \equiv T_{r}^{r} \quad, \quad P_{\theta} \equiv T_{\theta}^{\theta}=T_{\varphi}^{\varphi} .
$$

and $T_{\nu}^{\mu}=0$ for $\mu \neq \nu$.

We will for convenience introduce the following alternative form of the metric variables: 


$$
A^{2}(r)=\left(1-\frac{2 m(r)}{r}\right)^{-1} \quad, \quad N^{2}(r)=\exp [2 \nu(r)],
$$

Einstein's equations then read

$$
\begin{aligned}
\partial_{r} m & =4 \pi r^{2} \rho, \\
\partial_{r} \nu & =\frac{m(r)}{r^{2}}\left(1-\frac{2 m(r)}{r}\right)^{-1}\left(1+\frac{4 \pi r^{3} P_{r}}{m(r)}\right),
\end{aligned}
$$

The equation of hydrostatic equilibrium resulting from the conservation of the energy-momentum tensor $\nabla_{\nu} T^{\mu \nu}=0$ becomes

$$
\partial_{r} P_{r}=-\left(\partial_{r} \nu\right) \rho\left(1+\frac{P_{r}}{\rho}\right)-\frac{2}{r \rho}\left(P_{r}-P_{\theta}\right) .
$$

We note that the observations in spiral galaxies [17], yield $z_{D}=v \approx$ constant and $z_{D}>>z_{A}$. From these conditions and from (12) we obtain

$$
\frac{1}{N(r)} \frac{1}{\left[1-r \partial_{r} N(r) / N(r)\right]^{1 / 2}} \approx 1 \text {. }
$$

and

$$
v \equiv\left[r \partial_{r} N(r) / N\right]^{1 / 2},
$$

the value of $v$ roughly ranges from $10^{-4}$ to $10^{-3}$ depending of a particular spiral galaxy. The integration of (19) gives,

$$
N(r)=\left(\frac{r}{r_{g}}\right)^{v^{2}},
$$

where $r_{g}$ is constant.

Note that using (21) in Eqs. (17) and (18), we obtain a system of three equations [i.e., Eqs.(16)-(18)] for four unknowns (i.e, $m, \rho, P_{r}, P_{\theta}$ ). In the case of a perfect fluid, however, the four unknowns are reduced to three since $P_{r}=P_{\theta} \equiv p$. This therefore constrains the equation of state $p=p(\rho)$. On the other hand, for the case where the matter content is associated for example, with a scalar field, then $\rho, P_{r}$ and $P_{\theta}$ are not independent but are given in terms of the gradients of the field. This constrains the form of the scalar potential.

We will look for a solution that satisfies the Newtonian conditions,

$$
\begin{array}{r}
P_{r, \theta}<<\rho, \\
m(r)<<\frac{r}{2}, \\
4 \pi r^{3} P_{r}<<m(r) .
\end{array}
$$

Under these conditions the Eqs. (16)-(18) reduce to 


$$
\begin{aligned}
\partial_{r} m(r) & =4 \pi r^{2} \rho, \\
\partial_{r} \nu & =\frac{m(r)}{r^{2}}, \\
\partial_{r} P_{r} & =-\left(\partial_{r} \nu\right) \rho,
\end{aligned}
$$

using the expression (21) for $N$ in (26), (27) with $\nu=\ln [\mathrm{N}]$, and solving the system, we obtain

$$
m(r) \approx v^{2} r, \quad \rho(r) \approx \frac{v^{2}}{4 \pi r^{2}}, \quad P_{r} \approx \frac{v^{4}}{8 \pi r^{2}},
$$

The solution corresponds to the relation $P_{r} \approx v^{2} \rho / 2$ which looks somewhat peculiar. If we view this as the equation of state of a perfect fluid, in the case of an ideal gas we would conclude that its temperature $T$ is constant and proportional to $v^{2}$. The interpretation is that the dark matter represented by a perfect fluid is made of particles (ideal gas) that interact among themselves strongly enough to maintain thermal equilibrium but do not interact in the same way with ordinary matter or with photons. The idea is then that the dark matter temperature determines its density profile and the spacetime metric, and through this, the rotation curves of the stars in the galaxy. One of the problems of this type of models is the need to explain why the isothermal configuration of the fluid does not extend to the center of the galaxy.

Using Eqs. (21) and (28) in (1) we obtain the final result for the metric in this limit:

$$
d s^{2}=-\left(\frac{r}{r_{g}}\right)^{2 v^{2}} d t^{2}+(1-\alpha)^{-1} d r^{2}+r^{2}\left(d \theta^{2}+\sin ^{2} \theta d \varphi^{2}\right)
$$

where $\alpha=2 v^{2}$. We emphasize that this solution is only valid in the flat $\mathrm{RC}$ zone. We know that eventually this must be matched to a Robertson-Walker metric describing the universe or alternative we might use the asymptotically flat idealization for regions very far from the galaxy in question. To do so we must consider (29) as describing the spacetime geometry for $r<R_{0}$ and the Schwarzschild metric for $r>R_{0}$, where $R_{0}$ is the radius where the flat rotation curves end. The advantage of this approximation is that far from the galaxy the spacetime is Minkowskian a fact that facilitates, for example, the analysis of the propagation of light signals.

Matching continuously the two metrics at $R_{0}$ allows the determination of the integration constants:

$$
\begin{array}{cc}
d s^{2}=-(1-\alpha)\left(\frac{r}{R_{0}}\right)^{2 v^{2}} d t^{2}+(1-\alpha)^{-1} d r^{2}+r^{2}\left(d \theta^{2}+\sin ^{2} \theta d \varphi^{2}\right) & r<R_{0} \\
d s^{2}=-\left(1-\frac{2 M}{r}\right) d t^{2}+\left(1-\frac{2 M}{r}\right)^{-1} d r^{2}+r^{2}\left(d \theta^{2}+\sin ^{2} \theta d \varphi^{2}\right) & r>R_{0}
\end{array}
$$


where $M=\alpha R_{0} / 2$.

Here we are taking the view that the region of flat rotation curves, terminates in a narrow transition region where the behavior of the density changes from the $1 / r^{2}$ to a constant that for simplicity we take to be zero. The point being that in the limit in which the region is very narrow the metric will not change abruptly as we cross the region, but the derivative of the metric coefficients will experience a jump. It would be interesting to consider various alternatives for the details of the interpolating regions.

An alternative to the search of solutions satisfying the Newtonian conditions (22) - (24) is to start from the Eqs.. (16) - (18) without imposing the Newtonian approximation but assuming that the dark matter is represented by a perfect fluid. Then again, the use of (21) with $P_{r}=P_{\theta}$ allows the integration of $(16)-(18)$ as follows:

$$
m(r)=\frac{v^{2}}{2} \frac{\left(2-v^{2}\right)}{\left(1+2 v^{2}-v^{4}\right)} r, \rho(r)=\frac{v^{2}}{8 \pi} \frac{\left(2-v^{2}\right)}{\left(1+2 v^{2}-v^{4}\right) r^{2}}, \quad P_{r}=\frac{v^{4}}{8 \pi} \frac{1}{\left(1+2 v^{2}-v^{4}\right) r^{2}}
$$

which results in an "equation of state" $P_{r}=v^{2} \rho /\left(4-2 v^{2}\right)$. In practice $v^{2}<<1$, so we recover the solution (28).

At this point we can check whether the approximations we considered are self consistent. That is, we substitute (21) in the left hand side of (19) obtaining:

$$
\frac{1}{N(r)} \frac{1}{\left[1-r \partial_{r} N(r) / N(r)\right]^{1 / 2}}=\sqrt{\frac{1}{\left(1-v^{2}\right)(1-\alpha)}}\left(r / R_{0}\right)^{-v^{2}} .
$$

The difference between this expression and 1 must be negligible in comparison to $v$ which itself is of the order $10^{-3}-10^{-4}$. This requires $r / R_{0}$ to be neither to large nor to small. To get an estimate we use the approximation $X^{-v^{2}} \approx$ $1-v^{2} \ln (X)$ valid for $\left|v^{2} \ln (X)\right|<<1$. These requirements are then:

$$
\left|\ln \left(r / R_{0}\right)\right|<<v^{-2}
$$

Thus the approximations are self consistent as long as $-10^{6}<<\ln \left(r / R_{0}\right)<<$ $10^{6}$, which doesn't impose any practically relevant constraints for the case of the galaxies and the extent of the flat RC.

It is worth noting that the form of the metric that we have obtained differs from what would be naively expected:

$$
d s^{2}=-(1+2 \Phi) d t^{2}+(1+2 \Phi)^{-1} d r^{2}+r^{2}\left(d \theta^{2}+\sin ^{2} \theta d \varphi^{2}\right)
$$

with $\Phi$ representing the Newtonian potential. This form is often implicitly assumed (see [23]) and the fact that it is not appropriate for the region where the $\mathrm{RC}$ are flat lies at the core of the problems with the analysis of [35] (see 
[36]).

We also point out that if we assume that the flat RC extend indefinitely, the resulting spacetime is not asymptotically flat but rather is asymptotically flat but for a deficit angle (AFDA) [38]. In this context we stress that it is possible to consider such "idealized infinitely extended galaxies" as isolated objects in the framework of general relativity by replacing the asymptoticallyflat framework by the framework AFDA [38]. One might want to embark in such considerations since in fact the $\mathrm{RC}$ remain flat to the farthest distances that can be observed. On the other hand there is a natural way to estimate an upper bound for the cutoff of such behavior. The idea is to consider the point at which the decaying density profile associated with the galaxy becomes smaller than the average energy density of the universe. We call this bound $R_{M a x}^{U}$. The value for $R_{\text {Max }}^{U}$ is obtained by imposing the condition that the density at this point, provided by Eq. (28), is to coincide with the mean density of the universe:

$$
\rho\left(R_{M a x}^{U}\right) \approx \frac{v^{2}}{4 \pi\left(R_{\text {Max }}^{U}\right)^{2}}=\rho_{U}
$$

where $\rho_{U}$ is the mean density of the universe. Then we have

$$
R_{M a x}^{U}=\sqrt{\frac{v^{2}}{4 \pi \rho_{U, I}}}
$$

Now we introduce the value $\rho_{U}$ in terms of the dimensionless Hubble parameter $h$ defined as $H_{0} \equiv 100 h \mathrm{~km} /(\mathrm{sec} \mathrm{Mpc})$ and $\Omega_{U} \equiv \rho_{U} / \rho_{\text {crit }}$

$$
\rho_{U}=1.8791 \times 10^{-26} \Omega_{U} h^{2} \mathrm{~kg} / \mathrm{m}^{3}=2.78 \times 10^{11} \Omega_{U} h^{2} M_{\odot} / \mathrm{Mpc}^{3} .
$$

We obtain,

$$
R_{M a x}^{U}=2.45 \times 10^{6} \frac{v}{h} \Omega_{U}^{-1 / 2} \mathrm{Kpc}
$$

Taking $h=0.65$ and $v \approx\left(10^{-3}-10^{-4}\right)$, we have

$$
R_{\text {Max }}^{U}=3.77 \times\left(10^{3}-10^{2}\right) \Omega_{U}^{-1 / 2} \mathrm{Kpc} .
$$

Moreover, for $\Omega_{U}=1$,

$$
R_{M a x}^{U} \approx 3.77 \times\left(10^{3}-10^{2}\right) \mathrm{Kpc} .
$$


On the other hand, the measured flat regions are about $R_{0} \approx 2 R_{\text {opt }}$ where $R_{\text {opt }}$ is the radius encompassing 83 per cent of the total integrated light of the galaxy. We can take as a typical value $R_{0} \approx 30 \mathrm{Kpc}$, then $R_{0}<R_{\text {Max }}^{U}$.

\section{BENDING OF LIGHT IN THE DARK MATTER ZONE}

One of the ways we could in principle explore the issue of whether the flat $\mathrm{RC}$ are the result of some form of unknown matter or the result of the change in the dynamical laws that governs the motion of particles, would be by studying the light deflection by galaxies. In particular, by studying the deflection of photons passing through the region where the $\mathrm{RC}$ are flat. Let us thus consider a photon approaching the spiral galaxy from far distances. We will compute the bending of light assuming the metric that has been matched with an asymptotically flat exterior, i.e., Eq. (30).

The bending of the light results [9],

$$
\Delta \phi=2\left|\phi\left(r_{0}\right)-\phi_{\infty}\right|-\pi
$$

where $\phi_{\infty}$ is the incident direction and $r_{0}$ is the coordinate radius of closest approach to the center of the galaxy.

$$
\phi\left(r_{0}\right)-\phi_{\infty}=\int_{r_{0}}^{\infty} A(r)\left[\left(\frac{r}{r_{0}}\right)^{2} \frac{N^{2}\left(r_{0}\right)}{N^{2}(r)}-1\right]^{-1 / 2} \frac{d r}{r}
$$

The integral is split in two parts for the two domains of metric (30):

$$
\phi\left(r_{0}\right)-\phi_{\infty}=\int_{r_{0}}^{R_{0}} A(r)\left[\left(\frac{r}{r_{0}}\right)^{2} \frac{N^{2}\left(r_{0}\right)}{N^{2}(r)}-1\right]^{-1 / 2} \frac{d r}{r}+\int_{R_{0}}^{\infty} A(r)\left[\left(\frac{r}{r_{0}}\right)^{2} \frac{N^{2}\left(r_{0}\right)}{N^{2}(r)}-1\right]^{-1 / 2} \frac{d r}{r}
$$

The second integral is computed by expanding the integrand in powers of $M / r_{0}$ and $M / r$ [9] using (30) for $r>R_{0}$,

$$
\left[\left(\frac{r}{r_{0}}\right)^{2} \frac{N^{2}\left(r_{0}\right)}{N^{2}(r)}-1\right]=\left(\frac{r}{r_{0}}\right)^{2}\left[1+2 M\left(\frac{1}{r}-\frac{1}{r_{0}}\right)+\ldots .\right]=\left[\left(\frac{r}{r_{0}}\right)^{2}-1\right]\left[1-\frac{2 M r}{r_{0}\left(r+r_{0}\right)}+\ldots\right]
$$

and the results is 


$$
\begin{aligned}
& \int_{R_{0}}^{\infty} A(r)\left[\left(\frac{r}{r_{0}}\right)^{2} \frac{N^{2}\left(r_{0}\right)}{N^{2}(r)}-1\right]^{-1 / 2} \frac{d r}{r}=\int_{R_{0}}^{\infty} \frac{d r}{\left[\left(\frac{r}{r_{0}}\right)^{2}-1\right]^{1 / 2}}\left[1+\frac{M}{r}+\frac{M r}{r_{0}\left(r+r_{0}\right)}+\ldots . .\right. \\
= & \arcsin \left(\frac{r_{0}}{R_{0}}\right)+\frac{M}{r_{0}}\left[2-\left[1-\left(\frac{r_{0}}{R_{0}}\right)^{2}\right]^{1 / 2}-\left(\frac{R_{0}-r_{0}}{R_{0}+r_{0}}\right)^{1 / 2}\right]+\ldots .
\end{aligned}
$$

The first integral of (43) with (30) for $r<R_{0}$ gives

$$
\begin{aligned}
\int A(r)\left[\left(\frac{r}{r_{0}}\right)^{2} \frac{N^{2}\left(r_{0}\right)}{N^{2}(r)}-1\right]^{-1 / 2} \frac{d r}{r} & =(1-\alpha)^{-1 / 2} \int\left[\left(\frac{r}{r_{0}}\right)^{2\left(1-v^{2}\right)}-1\right]^{-1 / 2} \frac{d r}{r} \\
& =\frac{(1-\alpha)^{-1 / 2}}{\left(v^{2}-1\right)} \arctan \left[\left(\frac{r}{r_{0}}\right)^{2\left(1-v^{2}\right)}-1\right]^{-1 / 2} .
\end{aligned}
$$

Finally, using (45) and (46) in (41) and then using (42), the bending angle of light yields,

$$
\begin{aligned}
\Delta \phi= & \mid 2 \arcsin \left(\frac{r_{0}}{R_{0}}\right)+\frac{2 M}{r_{0}}\left[2-\left[1-\left(\frac{r_{0}}{R_{0}}\right)^{2}\right]^{1 / 2}-\left(\frac{R_{0}-r_{0}}{R_{0}+r_{0}}\right)^{1 / 2}\right]+ \\
& \frac{2(1-\alpha)^{-1 / 2}}{\left(v^{2}-1\right)}\left(\arctan \left[\left(\frac{R_{0}}{r_{0}}\right)^{2\left(1-v^{2}\right)}-1\right]^{-1 / 2}-\frac{\pi}{2}\right) \mid-\pi,
\end{aligned}
$$

where we took the limit

$$
\lim _{r \rightarrow r_{0}} \arctan \left[\left(\frac{r}{r_{0}}\right)^{2\left(1-v^{2}\right)}-1\right]^{-1 / 2}=\frac{\pi}{2}
$$

If we put $r_{0}=R_{0}$ in (47), we obtain the standard result for the Schwarzschild metric with mass $M=\alpha R_{0} / 2$ and with $\Delta \phi=\left(4 M / r_{0}\right)=4 \times 10^{-6}$. Fig.1] shows the bending angle of light $\Delta \phi$ as a function of the parameter $r_{0} / R_{0}$. If we take the impact parameter, $r_{0}$, to be in the range of the measured flat regions by neutral Hydrogen measurements (HI): $R_{\text {opt }} \leq r_{0} \leq 2 R_{\text {opt }}=R_{0}$, then we have $1 / 2 \leq r_{0} / R_{0} \leq 1$. In this case, the maximum value for $\Delta \phi$ is obtained for the value $r_{0} / R_{0}=1 / 2$. Recently, the investigations for determining the radius of dark matter halos have gone beyond the HI measurements using satellite galaxies [27] or the weak lensing of background galaxies by foreground dark halos [28]. From these measurements, halo radii of more than $200 \mathrm{Kpc}$ are inferred. For our galaxy $230 \mathrm{Kpc}$ [29] and from satellite galaxies of a set of spiral galaxies show more than $400 \mathrm{Kpc}[30]$. By taking $R_{0} \approx 230 \mathrm{Kpc}$, we would have $R_{\mathrm{opt}} \leq r_{0} \leq 15 R_{\mathrm{opt}}=R_{0}$ (where we have chosen $R_{\mathrm{opt}}=15 \mathrm{Kpc}$ ). In this case we would have $1 / 15 \leq r_{0} / R_{0} \leq 1$ and a value near to the maximum in the fig.1. It would be interesting to explore the possibility to have 
relevant observations in this context.

We discuss next two of the three simplest scenarios in which the dark matter corresponds to coherent scalar fields.

\section{SPONTANEOUS SCALARIZATION}

As we mention in the introduction, the phenomenon of spontaneous scalarization in compact objects (notably in neutron stars) that arise in a class of scalar tensor theories of gravity [24] is the other mechanism that allows the appearance of a non trivial scalar field in the absence of a direct coupling between the scalar field and ordinary matter. The general feature of these kind of theories is a scalar field coupled non-minimally to gravity which leads to an effective gravitational coupling which depends explicitly on the scalar field. The non-trivial scalar field configuration appears when the object is compact enough so that the energy of the configuration for a fixed baryon number is minimized through a change in the value of the effective gravitational constant. That is, for a fixed baryon number, the energy of the configuration with a scalar field is lower than the corresponding configuration in absence of a scalar field [25]. An heuristic interpretation that is confirmed by the numerical results shows that, from a Newtonian point of view, the relevant quantity to be minimized is the combination $G M$ instead of the total mass $M$. We observed that although the obvious additional contributions to GM [31] are both positive and thus increasing the value of $G M$, their effect is more than compensated by the reduction of the value of the contribution $G M_{\mathrm{bar}}$, which is the leading term in GM. Thus there appears a nontrivial configuration of the scalar field which is associated with the minimization (at fixed total baryon number) of the the value $G M$ [25].

Several problems arise if we want to use this mechanism to induce a non trivial configuration of a scalar field at the galactic scale. First, in the model studied so far we have seen that spontaneous scalarization occurs only if the object is compact enough, that is, if $G M / R \sim 1 / 2$ and needless to say that the galaxy as a whole does not satisfy this criteria (except perhaps at the center). If we assume that a large dense object lies at the center of the galaxy one would need some very unusual equation of state to overcome the standard limits on the mass of these objects associated with the requirement of stability against collapse. But even if we were to assume such an object, the scalar field associated with the phenomena of spontaneous scalarization falls as $1 / r$ (at least in the models considered so far) so it would not be relevant at the distances associated with the flat RC that lie at a distance of the order of kiloparsecs from the galactic center. Finally, the energy of the configurations with nontrivial configuration according to this phenomenon is smaller than that of the corresponding configuration in which the scalar field vanishes, thus the phenomenon seems to take us in the opposite direction as compared to 
what seems to be required to explain the additional attractive effect on the test stars in the galaxy. If we wanted to consider extended objects other that neutron stars, it is not even clear how to build a sufficiently dense object. The only possibility would seem to be boson stars [46] which now would act only as triggers of the spontaneous scalarization. These models would require to hypothesize two scalar fields, one providing the oscillating boson field of the boson star, and a second one providing the mechanism for spontaneous scalarization. On the other hand we must point out that although boson star masses are usually very small, when one introduces self-interactions their mass can be as large as $10^{27} \lambda^{1 / 2} M_{\odot}$ (for a scalar field mass $m \sim 10^{-5} \mathrm{eV}$ and a sufficiently large self-interaction constant $\lambda$ ) [42]. Still we face the problem associated with the rapid fall off of the energy density associated with the scalar field, which would go like $1 / r^{4}$.

The hope here would be to consider alternative forms of the nonminimal coupling, with the possible introduction of various forms of self interaction terms for the scalar field, that would not only lead to spontaneous scalarization but to a rather different fall off behavior of the scalar field. Nevertheless, as was already mentioned, there is one very serious problem remaining with this type of scenario, and it is the issue of black holes. There is at the present time mounting evidence that there is at the center of most galaxies a very massive black hole, and in view of the no hair theorems for scalar fields [19 21], it seems clear that the phenomena of spontaneous scalarization does not have an analogy when the compact objects are replaced by black holes. Thus in those galaxies the scalar field would relax to the trivial configuration and thus any explanation of the RC based on that phenomena would cease to be operative. There are, however, some small loopholes remaining in the black hole uniqueness theorems for the case of nonminimally coupled scalar fields which leave a ray of hope in this general direction, and which are currently under investigation [47.

For the case of massless bosons (massless complex scalar fields), a Newtonian analysis leads to flat RC 48]. Unfortunately, in that work, the author neglect to note that the $\mathrm{RC}$ are not directly observable but only inferred from the corresponding light shifts. As it turns out, in that model the "gravitational" red-shift would be very large to the point that by ignoring it, the author is ignoring effects of the same order of magnitude as the ones that are being considered. Moreover, the law of composition of velocities used there to reproduce the $\mathrm{RC}$ is not valid.

In the following section we analyze the case for the matter represented by global monopoles non-minimally coupled to gravity. 


\section{GLOBAL MONOPOLES}

We will now consider one example of what we feel is at this time the most promising class of models: nonminimally coupled global monopoles. The main results of this section have been reported in [34].

Particle physics models predict the formation of topological defects during phase transitions in the early universe. The mechanism argued for the formation of these is the spontaneous breaking of symmetry of the model under consideration leading to a manifold of degenerate vacua with nontrivial topology. Topological defects can be classified according to the topology of the vacuum manifold. If the manifold of equivalent vacua, $\mathcal{M}$, contains unshrinkable surfaces, $\pi_{2}(\mathcal{M}) \neq I$, then monopoles are formed. These can be classified into local and global monopoles depending on whether the symmetry broken is local or global. In the first case (gauge monopoles) the monopole configuration has finite energy concentrated in a small core and produces an asymptotically flat spacetime, while in the second case, the global-monopole configuration has a linearly divergent energy due to the long range NambuGoldstone field with energy density decreasing with the distance as $r^{-2}$. As we have mentioned, this behavior is very appealing in view of the fact that this is precisely what seems to be required in a naive picture to provide a natural explanation for the flatness of the RC.

It was shown by Barriola and Vilenkin [32] that this linearly divergent "mass" has, at large distances, an effect analogous to that of a deficit solid angle $\alpha$ plus that of a tiny mass associated to the core of the monopole. Then, assuming the existence of a global monopole in a typical galaxy the total Newtonian mass contribution of the portion of the global monopole contained within $r_{\text {gal }}$ (with $r_{\text {gal }} \approx 15 \mathrm{Kpc}$ ) is found to be $M \sim \alpha r_{\text {gal }} / 2 \approx 10^{69} \mathrm{GeV}$, where we took a typical grand unified value $\eta \approx 10^{16} \mathrm{GeV}$, and where $\alpha=8 \pi G \eta^{2}$. This estimate turns out to be 10 times the total mass due to the contribution of $10^{11}$ solar-mass in a typical galaxy (i.e., $M_{\text {stars }} \approx 10^{68} \mathrm{GeV}$ ). These numbers are again what is needed to account for the observations. Finally, if we assume that the field of the monopole extends on average a distance of ten galactic radii from the galaxy where the configuration presumably coincides with that of the monopole centered in the neighbouring galaxy, then $M \approx 10^{70} \mathrm{GeV}$, which is 100 times that of the galaxy. This value leads to a contribution of the monopole to the total average density in the universe, which is of the order of magnitude predicted by the standard inflationary scenarios. Actually, it is the reversed argument that helps to place upper bounds on the density number of global monopoles present in the universe [37]. On the other hand, Harari and Loustó [33], showed that the small effective mass $m_{\text {core }} \approx 0.8 \alpha$ is in fact negative and produces a repulsive potential. They studied the motion of test particles in the spacetime of a global monopole concluding that there are no bound orbits. This result led thus to the unavoidable conclusion that 
minimally coupled global monopoles are not good candidates to explain the $\mathrm{RC}$ despite the suggestive numbers and features considered above.

Another problem is the fact that the monopole configuration is rather unique, in the sense that it is basically independent of the ordinary matter content in the corresponding galaxy, which conflicts with the fact that there is a rather large range of galactic masses for which the dark matter component is about ten times more massive than the ordinary matter component [39].

Recently, we have shown [34], that coupling global monopoles non-minimally to gravity is possible to avoid the most unwanted features of the minimal case, notably, the lack of bound orbits, and the universality of the monopole configuration.

Specifically we considered a theory of a triplet of scalar fields $\phi^{a}, a=1,2,3$, non-minimally coupled (NMC) to gravity with global $\mathrm{O}(3)$ symmetry which is broken spontaneously to $\mathrm{U}(1)$. The simplest model of this kind is described by the Lagrangian

$$
\mathcal{L}=\sqrt{-g}\left[\frac{1}{16 \pi} R+F\left(R, \phi^{a} \phi_{a}\right)\right]-\sqrt{-g}\left[\frac{1}{2}\left(\nabla \phi^{a}\right)^{2}+V\left(\phi^{a} \phi_{a}\right)\right],
$$

where $V\left(\phi^{a} \phi_{a}\right)$ is the usual Mexican hat potential $V\left(\phi^{a} \phi_{a}\right)=\frac{\lambda}{4}\left(\phi^{a} \phi_{a}-\eta^{2}\right)^{2}$.

Equation (49) shows that the introduction of the coupling term is equivalent to consider an effective potential

$$
V\left(\phi^{a} \phi_{a}\right)_{\mathrm{eff}}=V\left(\phi^{a} \phi_{a}\right)-F\left(R, \phi^{a} \phi_{a}\right)
$$

which explicitly depends on the matter content through $R$, and thus the content of ordinary matter of the galaxy affects the location of the minima. This feature can thus help to avoid the scenario where the monopole configuration is universal, and opens the possibility to recover the correlation between the masses in the dark and ordinary matter components of the galaxy.

In the following we show in detail how the non-minimal coupling leads to the existence of bound orbits. We will focus on the case where $F\left(R, \phi^{a} \phi_{a}\right)=$ $\left(\xi \phi^{a} \phi_{a}\right) R$, where $\xi$ is the NMC constant. The gravitational field equations following from the Lagrangian (49) can be written as

$$
R^{\mu \nu}-\frac{1}{2} g^{\mu \nu} R=8 \pi G_{0} T_{\text {eff }}^{\mu \nu}
$$

where

$$
\begin{aligned}
& T_{\mathrm{eff}}^{\mu \nu}=\frac{G_{\mathrm{eff}}}{G_{0}}\left(4 \xi T_{\xi}^{\mu \nu}+T_{\mathrm{sf}}^{\mu \nu}\right), \\
& T_{\xi}^{\mu \nu}=\nabla^{\mu}\left(\phi^{a} \nabla^{\nu} \phi_{a}\right)-g^{\mu \nu} \nabla_{\lambda}\left(\phi^{a} \nabla^{\lambda} \phi_{a}\right), \\
& T_{\mathrm{sf}}^{\mu \nu}=\nabla^{\mu} \phi^{a} \nabla^{\nu} \phi_{a}-g^{\mu \nu}\left[\frac{1}{2}\left(\nabla \phi^{a}\right)^{2}+V\left(\phi^{a} \phi_{a}\right)\right] .
\end{aligned}
$$


The equation of motion for the scalar fields is,

$$
\square \phi^{a}+2 \xi \phi^{a} R=\frac{\partial V\left(\phi^{b} \phi_{b}\right)}{\partial \phi_{a}} .
$$

We will only consider a metric describing spherical and static space-times (1) and study solutions of the gravitational and scalar fields equations describing global monopole configurations and the resulting space-time. Owing to the complexity of the resulting equations, we will perform a numerical analysis in terms of the following variables:

$$
\begin{aligned}
\nu(r) & =\ln [N(r)], \\
\tilde{\nu}(r) & =\nu(r)-\nu(0), \\
A(r) & =\left(1-\alpha-\frac{2 G_{0} m(r)}{r}\right)^{-1 / 2} .
\end{aligned}
$$

where

$$
\alpha=\frac{\Delta}{1+2 \xi \Delta}, \quad \Delta=8 \pi G_{0} \eta^{2}
$$

The relevant Einstein equations take then the following form

$$
\begin{aligned}
& \frac{\partial m}{\partial r}=4 \pi r^{2} E-\frac{\alpha}{2 G_{0}}, \\
& \frac{\partial \nu}{\partial r}=A^{2}\left\{\frac{G_{0} m}{r^{2}}+\frac{\alpha}{2 r}+4 \pi r G_{0} T_{\text {eff } r}^{r}\right\} .
\end{aligned}
$$

where

$$
E=N^{2} T_{\mathrm{eff}}^{t t}
$$

is the effective total energy density.

On the other hand, the Klein-Gordon equation can be written directly in terms of the energy momentum of the scalar fields:

$$
\square \phi^{a}=-16 \pi \xi \phi^{a} G_{0}(E-S)+\frac{\partial V\left(\phi^{b} \phi_{b}\right)}{\partial \phi_{a}} .
$$

where

$$
S=T_{\text {eff } i}^{i}
$$

is the trace of the "spatial part" of $T_{\text {eff }}^{\mu \nu}$, which plays the role of an effective pressure.

In the coordinates (1) this equation reads 


$$
\begin{aligned}
\frac{\partial^{2} \phi^{a}}{\partial r^{2}}= & -\left[\frac{2}{r}+\frac{\partial \nu}{\partial r}-\left(1-\alpha-\frac{2 G_{0} m}{r}\right)^{-1}\left(4 \pi G_{0} r E-\frac{G_{0} m}{r^{2}}-\frac{\alpha}{2 r}\right)\right] \frac{\partial \phi^{a}}{\partial r} \\
& +\left(1-\alpha-\frac{2 G_{0} m}{r}\right)^{-1}\left[\frac{\partial V\left(\phi^{b} \phi_{b}\right)}{\partial \phi_{a}}-16 \pi \xi \phi^{a} G_{0}(E-S)\right] \\
& -\frac{1}{r^{2}}\left(1-\alpha-\frac{2 G_{0} m}{r}\right)^{-1}\left[\frac{\partial^{2} \phi^{a}}{\partial \theta^{2}}+\frac{\cos \theta}{\sin \theta} \frac{\partial \phi^{a}}{\partial \theta}+\frac{1}{\sin ^{2} \theta} \frac{\partial^{2} \phi^{a}}{\partial \varphi^{2}}\right] .
\end{aligned}
$$

The ansatz for a monopole configuration is

$$
\phi^{a}=\eta f(r) \frac{x^{a}}{r},
$$

with $x^{a} x^{a}=r^{2}$, so that a monopole solution is found if $f \rightarrow 1$ at spatial infinity (i.e., $\left.\left\|\phi^{a}\right\| \rightarrow \eta\right)$.

It is clear from the Eqs. (52 54), that the intermediary variables $E$ and $S$ [see Eqs.(62), (64)] involve second order derivatives of the scalar field. However, we can eliminate such a terms from the gravitational field equations with the help of the ansatz for the monopole field and of the Klein-Gordon equation, and obtain "sources" containing at most first order derivatives of the scalar field. We also introduce the following dimensionless quantities

$$
\begin{aligned}
\tilde{r} & :=r \cdot \eta \lambda^{1 / 2}, \\
\tilde{m} & :=m \cdot G_{0} \eta \lambda^{1 / 2}, \\
\tilde{\rho} & :=\rho \cdot \frac{G_{0}}{\eta^{2} \lambda}, \\
\tilde{p} & :=p \cdot \frac{G_{0}}{\eta^{2} \lambda}, \\
\tilde{\phi}^{a} & :=\frac{\phi^{a}}{\eta}, \\
\frac{\phi^{a}}{\eta} & :=f(r) \frac{x^{a}}{r}, \\
\tilde{V}\left(\tilde{\phi}^{a} \phi_{a}\right) & :=V\left(\phi^{a} \phi_{a}\right) \cdot \frac{G_{0}}{\eta^{2} \lambda}, \\
\Delta & :=8 \pi G_{0} \eta^{2}, \\
\alpha & :=\frac{\Delta}{1+2 \xi \Delta}, \\
\tilde{G}_{\mathrm{eff}} & :=\frac{1}{1+2 \xi \Delta f^{2}},
\end{aligned}
$$

then the final form of the equations to be analyzed numerically is :

$$
\begin{aligned}
& \partial_{\tilde{r}} \tilde{m}=4 \pi \tilde{r}^{2} \tilde{E}-\frac{\alpha}{2}, \\
& \partial_{\tilde{r}} \tilde{\nu}=\frac{A^{2}}{1+2 \xi \Delta \tilde{r} f\left(\partial_{\tilde{r}} f\right) \tilde{G}_{\text {eff }}}\left\{\frac{\alpha}{2 \tilde{r}}+\frac{\tilde{m}}{\tilde{r}^{2}}+\frac{\Delta}{2} \tilde{r} \tilde{G}_{\text {eff }}\left[\frac{1}{2 A^{2}}\left(\partial_{\tilde{r}} f\right)^{2}-\frac{\left(f^{2}-1\right)^{2}}{4}-\frac{f^{2}}{\tilde{r}^{2}}-\frac{8 \xi f\left(\partial_{\tilde{r}} f\right)}{\tilde{r} A^{2}}\right]\right\},
\end{aligned}
$$




$$
\begin{aligned}
\partial_{\tilde{r} \tilde{r}} f= & -\left[\frac{2}{\tilde{r}}+\partial_{\tilde{r}} \tilde{\nu}-\left(1-\alpha-\frac{2 \tilde{m}}{\tilde{r}}\right)^{-1}\left(4 \pi \tilde{r} \tilde{E}-\frac{\alpha}{2 \tilde{r}}-\frac{\tilde{m}}{\tilde{r}^{2}}\right)\right]\left(\partial_{\tilde{r}} f\right) \\
& +\left(1-\alpha-\frac{2 \tilde{m}}{\tilde{r}}\right)^{-1}\left[f\left(f^{2}-1\right)+\frac{2 f}{\tilde{r}^{2}}-16 \pi \xi f(\tilde{E}-\tilde{S})\right]
\end{aligned}
$$

where

$$
\begin{gathered}
\tilde{E}-\tilde{S}=\frac{\Delta \tilde{G}_{\text {eff }}}{8 \pi\left(1+24 \Delta \xi^{2} f^{2} \tilde{G}_{\text {eff }}\right)}\left[\left(\frac{1}{A^{2}}\left(\partial_{\tilde{r}} f\right)^{2}+\frac{2 f^{2}}{\tilde{r}^{2}}\right)(1+12 \xi)+\left(f^{2}-1\right)^{2}+12 \xi f^{2}\left(f^{2}-1\right)\right], \\
\tilde{E}=\frac{\Delta \tilde{G}_{\text {eff }}}{8 \pi\left(1+24 \Delta \xi^{2} f^{2} \tilde{G}_{\text {eff }}\right)}\left[-\frac{4 \xi f\left(\partial_{\tilde{r}} f\right)\left(\partial_{\tilde{r}} \tilde{\nu}\right)}{A^{2}}\left(1+24 \Delta \xi^{2} f^{2} \tilde{G}_{\text {eff }}\right)+4 \xi f^{2}\left(f^{2}-1\right)\right. \\
+\left(\frac{1}{2 A^{2}}\left(\partial_{\tilde{r}} f\right)^{2}+\frac{f^{2}}{\tilde{r}^{2}}\right)\left(1+8 \xi+8 \Delta \xi^{2} f^{2} \tilde{G}_{\text {eff }}\right) \\
\left.+\frac{\left(f^{2}-1\right)^{2}}{4}\left(1-8 \Delta \xi^{2} f^{2} \tilde{G}_{\text {eff }}\right)\right]
\end{gathered}
$$

Here $\tilde{E}$ and $\tilde{S}$ are dimensionless as in Eq. (69).

We note now that the sources of the differential equations contain only first order derivatives of the field variables and are thus suitable for numerical integration with a Runge-Kutta algorithm.

\section{A. Asymptotic expansions and boundary conditions}

Let us discuss the asymptotic behavior of global monopoles at the origin and spatial infinity in order to find the boundary conditions for the numerical integration. The regularity condition at $r=0$ on the metric requires

$$
\tilde{m}(0)=0, \partial_{\tilde{r}} \tilde{m}(0)=-\alpha / 2 .
$$

The boundary condition on $\tilde{\nu}(r)$ is by definition

$$
\tilde{\nu}(0) \equiv 0 \text {. }
$$

The boundary condition on the scalar field at $r=0$ is (false vacuum),

$$
f(0)=0 \text {. }
$$

Then one finds the expansions of the functions $\tilde{m}(\tilde{r}), f(\tilde{r})$ and $\tilde{\nu}(\tilde{r})$, at $r=0$,

$$
\begin{gathered}
\tilde{m}(\tilde{r})=-\frac{\alpha}{2} \tilde{r}+\frac{\Delta}{12}\left[\frac{1}{2}+3(8 \xi+1) f_{c}^{2}\right] \tilde{r}^{3}+O\left(\tilde{r}^{4}\right) \\
f(\tilde{r})=f_{c} \tilde{r}+O\left(\tilde{r}^{3}\right) \\
\tilde{\nu}(\tilde{r})=-\frac{\Delta}{24}(1+24 \xi) f_{c}^{2} \tilde{r}^{2}+O\left(\tilde{r}^{3}\right)
\end{gathered}
$$


where $f_{c}$ is determinated by the boundary conditions at spatial infinity.

Let define $M=\tilde{m}(\infty)$. As we mentioned, we consider monopole configurations (i.e., $f \rightarrow 1$ at spatial infinity: true vacuum). Then we have the asymptotic expansions at spatial infinity,

$$
\begin{gathered}
\tilde{m}(\tilde{r})=M+\frac{\alpha}{2 \tilde{r} \Delta}\left[\frac{\alpha^{2}}{\Delta}+\frac{8 \xi(1-\alpha)}{\alpha}\right]+O\left(\tilde{r}^{-2}\right), \\
f(\tilde{r})=1-\frac{1}{(1+2 \xi \Delta) \tilde{r}^{2}}+O\left(\tilde{r}^{-4}\right), \\
\tilde{\nu}(\tilde{r})=\tilde{\nu}(\infty)-\frac{M}{(1-\alpha) \tilde{r}}+O\left(\tilde{r}^{-2}\right) .
\end{gathered}
$$

We will impose for the asymptotic behavior of the metric, the standard asymptotically-flat-but-for-a-deficit-angle $\alpha$ spacetime (S.A.F.D.A $\alpha$ ) (see [38]) and therefore the boundary condition on the function $\nu(\tilde{r})$ at spatial infinity is

$$
\nu(\infty)=\frac{1}{2} \ln (1-\alpha) .
$$

The integration of the equations is performed by specifying the regularity condition on the scalar field at $r=0$,

$$
\partial_{\tilde{r}} f(0)=f_{c} .
$$

The value $f_{c}$ cannot be arbitrary, but must be so that $f$ satisfy the appropriate boundary conditions at spatial infinity. This is enforced by the use of a standard shooting method 41].

We can compute the solution by integrating the equations in one step, from $r=0$ to a radius which is chosen conveniently so that $f=1$ with a certain degree of approximation. The physical lapse $N(r)=e^{\nu}$ at $r=0$ is calculated at the end of the numerical integration from (57) and (91)

$$
\nu(0)=\frac{1}{2} \ln (1-\alpha)-\tilde{\nu}_{\infty},
$$

where the value $\tilde{\nu}_{\infty}$ is obtained from the numerical integration. This ensures that at spatial infinity we recover the S.A.F.D.A $\alpha$ spacetime.

We note that the ADM mass of the configurations of global monopoles, (see [38] for a rigorous definition of the ADM mass for the case of spacetimes with a deficit angle) can be easily computed from the integral

$$
M_{\mathrm{ADM} \alpha}=M=\lim _{r \rightarrow \infty} m(r)=\int_{0}^{\infty}\left(4 \pi r^{2} E(r)-\frac{\alpha}{2}\right) d r .
$$




\section{B. Results.}

In order to obtain static configurations, we must impose the condition $0<\alpha<1$ for the deficit angle. The behavior of the deficit angle $\alpha$ when $\Delta \sim 0$ is,

$$
\alpha \sim \Delta-2 \xi \Delta^{2}+O\left(\Delta^{3}\right)
$$

Case $\xi>1 / 2$. The range allowed for the breaking scale is $\Delta \in[0, \infty)$ and for the deficit angle $\alpha \in\left[0, \frac{1}{2 \xi}\right)$. This is consistent with the condition $\alpha<1$. Figures 2-1, shows numerical solutions for the case $\xi=2$ satisfying the required boundary conditions.

Case $\xi \leq 1 / 2$. The range permitted for the breaking scale is $\Delta \in[0,1 /(1-$ $2 \xi \alpha)]$ and for the deficit angle $\alpha \in[0,1]$. In this case the static configurations cease to exist when $\Delta>1 /(1-2 \xi \alpha)$.

Figures 2-4, shows numerical solutions for the cases $\xi=0.3$ and $\xi=0$ (minimal coupling case) satisfying the required boundary conditions.

In the minimal coupling case Harari \& Loustó [33] estimated analytically the mass of the monopole as $M \sim-2 \alpha / 3$, and the size of the core $\tilde{\delta} \sim 2$. Then numerically showed that $M \sim-0.75 \alpha$. That is, they showed that the ratio $M / \alpha$ is practically insensitive to $\alpha$ (see figs. (5).66).

In the nonminimal coupling case, for a given $\xi$ the situation is similar in that the ratio $M / \alpha$ is practically insensitive to $\alpha$ for $\alpha \leq 0.01$. Actually, in this range of small $\alpha$, that ratio depends weakly on the value of $\xi$. However, for $\alpha>0.01$ the ratio depends strongly on $\xi$ and $\alpha$ [cf. figs. 0 , 6]. This can be seen by performing an analysis similar to the one of [33] but assuming the following approximation

$$
f= \begin{cases}f_{c} \tilde{r} & \text { if } \tilde{r}<\tilde{\delta} \\ 1-\frac{1}{(1+2 \xi \Delta) \tilde{r}^{2}} & \text { if } \tilde{r}>\tilde{\delta}\end{cases}
$$

and for the function $\tilde{m}(\tilde{r})$

$$
\tilde{m}= \begin{cases}-\frac{\alpha}{2} \tilde{r}+\frac{\Delta}{24} \tilde{r}^{3} & \text { if } \tilde{r}<\tilde{\delta} \\ M & \text { if } \tilde{r}>\tilde{\delta}\end{cases}
$$

matching continuously the function $\tilde{m}$ and its derivative at $\tilde{r}=\tilde{\delta}$, we obtain,

$$
\begin{aligned}
M & =-\frac{2}{3} \frac{\Delta}{(1+2 \xi \Delta)^{3 / 2}}=-\frac{2}{3} \alpha(1+2 \xi \Delta)^{-1 / 2} \\
\tilde{\delta} & =\frac{2}{(1+2 \xi \Delta)^{1 / 2}}=2(1-2 \xi \alpha)^{1 / 2}
\end{aligned}
$$


where we have used the relation $\Delta=\frac{\alpha}{(1-2 \xi \alpha)}$. For the case $\xi=0$, we reproduce for $M$ and $\tilde{\delta}$ the values estimated analytically by Harari \& Loustó [33]. We can see that in the case $\xi>1 / 2$, we have $\Delta \in[0, \infty)$ and $\alpha \in\left[0, \frac{1}{2 \xi}\right)$ and therefore $M \rightarrow 0$ when $\alpha \rightarrow \frac{1}{2 \xi}$, these is confirmed numerically for the value $\xi=2[\mathrm{cf}$. fig. 6 ]. Now we match continuously the function $f$ at $\tilde{r}=\tilde{\delta}$ and we obtain

$$
f_{c}=\frac{2}{3 \sqrt{3}}(1+2 \xi \Delta)^{1 / 2}=\frac{2}{3 \sqrt{3}}(1-2 \xi \alpha)^{-1 / 2}
$$

then when $\alpha \rightarrow \frac{1}{2 \xi}$ we have that the value of $f_{c}$ diverges, this is checked out numerically for the value $\xi=2$ [cf. fig.7].

\section{Geodesic motion in the spacetime of a global monopole.}

In order to analyze the geodesic motion of test particles in the spacetime generated by a global monopole we consider Eq.(5). In this case $N_{D}=(1-\alpha)^{1 / 2}$ and the remaining gravitational potentials are given numerically. Figure 8 shows the effective potential for $\xi=-2$ and for different values of the other parameters; here we note the existence of a potential well and a non trivial minimum and thus the existence of stable circular orbits.

For $\xi>0$ the effective potential (5) does not exhibit maxima or minima. An heuristic analysis that helps to understand the appearance of extrema of $V_{\text {eff }}$ when $\xi<0$ and their absence when $\xi>0$, is the following. We have that, $\partial_{r} V_{e f f}=2 N^{2}(r)\left(-L^{2} / r^{3}+L^{2} \partial_{r} \nu / r^{2}+\partial_{r} \nu\right)$. So for $\partial_{r} V_{e f f}=0$ and then to find extrema, notably minima, it is necessary that $\partial_{r} \nu>0$ (or equivalently $\left.\partial_{r} N>0\right)$. This means that $N(r)$ should have an increasing behavior as a function of $r$ at least in some region away from the origin. Eq. (78) provides the sign for the slope of $N$. Near the origin $f \sim f_{c} r$ and then $\partial_{r} \nu \sim-(\Delta / 12)(1+24 \xi) r f_{c}^{2}+O\left(r^{3}\right)$. For $\partial_{r} \nu>0, \xi$ must be negative enough so that the coefficient $(1+24 \xi)$ is negative [cf. Fig.9]. On the other hand, for $\xi \geq 0, \partial_{r} \nu<0$ and therefore $V_{\text {eff }}$ has no minima (no bound orbits) [cf. Figs. [4, 10]. This heuristic argument is confirmed by the rigorous numerical analysis from which the critical value $\xi_{\text {crit }}$ that allows the existence of bound orbits is found to be $\xi_{\text {crit }} \lesssim-0.15$.

Moreover, for $L=0$ which corresponds to $V_{\text {eff }} \equiv N^{2}$, a peculiar situation occurs in the cases where $V_{\text {eff }}$ has extrema, notably a maxima (e.g., for $\xi=-2$ ). The maxima and minima of $V_{\text {eff }}$ will correspond to the locus of unstable and stable stationary points where test particles are static (i.e., where particles do not feel any gravitational field). As seen from fig.9, we 
appreciate that at the origin $r=0$, test particles can be at rest in stable equilibrium, while at $r_{\max }$ where $V_{\text {eff }}$ is maximum test particles can be in unstable static equilibrium. In other words, $r_{\max }$ separates two regions: one attractive and other repulsive [cf. the $N(r)$ profile from Fig.9]. This strange behavior contrast dramatically from the cases of "conventional" gravitational sources like stars, planets, etc. where test particles are always attracted towards the source and where there are "no trivial" points at which they can remain static. In the case of minimally coupled global monopoles test particles are always repelled.

Since bound orbit exists in the spacetime generated by nonminimal global monopole with suitable $\xi$, we can compute the corresponding shift $z_{D}$ from Eq.(12) using the numerical solutions for $N$ and compare with the RC of spiral galaxies. Figure 11 depicts $z_{+}$(dashed line), $z_{-}$(solid line) and $z_{D}$ (dash-dotted line) as functions of $\tilde{r}$ for the cases $\alpha=0.43$ (left panel) and $\alpha=0.125$ (right panel) respectively. We note that even for this very simple model the figures that would correspond to the rotation curves contain a relatively "flat region" within the values of $r$ corresponding to stable orbits (i.e. the behavior of $z_{D}$ near its maximum).

From these figures it is interesting to note that $z_{+}$that in principle is associated with a blue-shift, does not always correspond to a blue-shift, since there is a value $r_{b}$ that separates $z_{+}$of being positive (blue-shift) or negative (red-shift). This is easy to understand since the value $z_{+}$arise from a competition between the gravitational red-shift and the kinematical blueshift. For slow particles (i.e, particles orbiting at "small" $r$ ) moving in the same direction as the emitted light, the gravitational barrier dominates over the positive contribution of the kinematical effects, and thus the frequency of emitted light has an overall attenuation. At orbits with radius $r_{b}$, particles are fast enough for the kinematical blue-shift to cancel the gravitational redshift resulting in $z_{+}=0$. For example, from (11) and for $v<<1$, it turns that $z_{+}<0$ if $v<1-N(r) /(1-\alpha)^{1 / 2}$. We note that in the non-minimal coupling case $(1-\alpha)^{1 / 2}>N(r)$ in the regions where bound orbits exist. For larger values of $r, z_{+}$reaches a maximum and then starts decreasing since the gravitational barrier becomes larger (see Fig.8) while the tangential velocity $\left.v\left[v=\left(r \partial_{r} N / N\right)^{1 / 2}\right)\right]$ becomes smaller until reaching zero at the radius $r_{\max }$ where $N$ is maximum (see Fig.12).

Concerning $z_{-}$, this quantity is generically negative (i.e., it corresponds to a true red-shift). For instance, when $v<<1$, then $z_{-} \approx 1-(1-\alpha)^{1 / 2}(1+v) / N$ so $z_{-}<0$ if $(1-\alpha)^{1 / 2}(1+v) / N>1$. This condition holds in most of the region of bound orbits since then $N<(1-\alpha)^{1 / 2}$ and $v \neq 0$ (see Figs.9). However, moving away from the origin $v \rightarrow 0$ and $N$ grows to a maximum value where $v=0$ and $z_{-}=z_{+}=1-(1-\alpha)^{1 / 2} / N$ which can be positive. For the cases (values of $\xi$ ) giving rise to bound orbits, it seems that $N$ always has a global maximum and then $N_{\max }>(1-\alpha)^{1 / 2}$. Therefore $z_{ \pm}>0$ at 
$r_{\text {max }}$. In fact the region of $r$ where $z_{-} \geq 0$ is very narrow and corresponds to $(1-\alpha)^{1 / 2}(1+v) / N \leq 1$ [practically unseen at the scales of Fig.(11); this corresponds to orbits of small angular momentum].

To conclude this section, we mention that although the nonminimal global monopole model can repair the two main objections posed on the minimal model (namely, the bound orbit and the correlation between luminous and dark matter problems), there are still several improvements to perform in order that the quantitative predictions of this model fit reasonably well with the astrophysical data. Therefore, it is still very premature for any claim on this model as a realistic candidate for explaining the galactic dark matter and the corresponding rotation curves. The fact that the model is fully consistent in what regards the mathematical analysis (no singularities, no ad hoc prescriptions for the "tangential velocities" or for the metric), in addition to the numerical coincidences mentioned at the beginning of the section, provides some hope for pursuing a much more detailed study along this direction.

\section{CONCLUSIONS}

The galactic rotation curves continue to pose a challenge to present day physics as one would want to understand not only the nature of the dark matter that is associated with them but also the reason behind their universality (i.e., why is it distributed within a galaxy in a way that leads to almost flat rotation curves ?, and why is the amount of dark matter present in a galaxy so well correlated with the luminous matter ? [16,50]).

Models based in ordinary physical objects could already be facing problems (depending on the exact value of the Hubble constant [10]) in view of the bounds that big bang nucleosynthesis impose on the baryon content of the universe.

Models based on particle physics are the most commonly considered (usually within a Newtonian scheme) but they need to address the nature and the distribution problems separately, leading to a larger number of hypothesis and surprinsing coincidences [11].

In view of the recent cosmological measurements and the theories that have been put forward to explain them [49], one is naturally lead to consider alternative models based in the introduction of long range coherent fields 23. In this work, we have given a review of various types of approaches to these questions indicating in each case the problems and advantages.

We have argued that so far the most promising and simple approach would involve global monopoles with some sort of nonminimal coupling to gravity. This remains for the future to establish how far can this sort of ideas be pushed towards the goal of making a realistic and compelling model for the dynamics and evolution of galaxies. In particular any such model must also be studied in the context of cosmological perturbations, large scale structure and the CMB. In this regard we should point out that the simplest models of 
topological defects as seeds for structure formation seem to be incompatible with the acoustic peak in the CMB anisotropies detected by Boomerang and Maxima 51]. However, all these studies have considered the simplest minimally coupled models and it is unclear how would the models of the type being analyzed here behave in this respect. Finally, we should mention that the currently favored cosmological scenarios require at least two hypothetical components: the Cold Dark Matter (usually in the form of WIMPS) necessary for the structure growth and the dark matter in galaxies and clusters, and the cosmological constant $\Lambda$ which provides the closure density (as required by inflation) as well as the repulsive component that seems to be required in order to account for the observations of the luminosity-distance of high red shift (type Ia) supernovae [52]. The fact that the non-minimally coupled monopoles exhibit both an attractive regime at short distances and a repulsive regime at large distances leads us to speculate whether these type of models can be used to explain the two aspects of the unobserved energy content of the universe in terms of a single hypothetical component. Needless is to say that all these aspects will require intense further exploration, which we hope to undertake in the near future.

\section{Acknowledgments}

U.N. is supported by a CONACyT postdoctoral fellowship grant 990490; M.S. and D.S. acknowledge partial support from DGAPA-UNAM Project No. IN121298 and from CONACyT Projects 32551-E and 32272-E. Authors thank the supercomputing department of DGSCA-UNAM. 


\section{REFERENCES}

[1] V.C. Rubin, Science 220, 1339 (1983)

[2] M. S. Turner, Phys. Scr. T36, 167 (1991).

[3] J. R. Primack, Proceedings of the International School of Physics "Enrico Fermi", vol. 92, ed. N. Cabibbo. Bologna: Italian Physical Society, (1987).

[4] J. Wess, and B. Zumino, Nucl. Phys. B70, 39 (1974).

[5] E. W. Kolb, and M. S. Turner, The Early Universe, Addison Wesley, Redwood City, California, 1990

[6] B. J. Carr, J. R. Bond, W. D. Arnett, Astrophys. J. 277, 445 (1984).

[7] C. Alcock, et al., Astrophys. J. 486, 697 (1997).

[8] P. J. E. Peebles, Principles of Physical Cosmology, Princeton University Press, Princeton, (1993).

[9] Steven Weinberg, Gravitation and Cosmology: Priciples and Applications of the General Theory of Relativity (Jonh Wiley and Sons, Inc, 1972).

[10] C. J. Copi, D. N. Schramm and B. M. Tinsley, Science 267, 192 (1995).

[11] J. A. Sellwood,and A. Kosowsky, astro-ph/0009074.

[12] A. Finzi, Mon. Not. Roy. Astron. Soc. 127, 21 (1963).

[13] R. H. Sanders, Astron. Astrophys. 136, L21 (1984).

[14] M. Milgrom, Astrophys J. 270, 365 (1983).

15

[15] P. D. Mannheim, Astrophys J. 479, 659 (1997); ibid, ApJ, 419, 150 (1993).

[16] M. Persic, P. Salucci, F. Stel, M.N.R.A.S. 281, 271996.

[17] V. C. Rubin, W. K. Ford, and N. Thonnard, Astrophys. J. 238, 471 (1980); V. C. Rubin, W. K. Ford, N. Thonnard, and D. Burstein, ibid. 261, 439 (1982); 289, 81 (1985).

[18] V. B. Braginskii, V. I. Panov, Zh. Eksp. Teor. Fiz. 61, 873 (1971).

[19] M. Heusler, J. Math. Phys. 33, 3497 (1992); ibid Class. Quant. Grav. 12, 779 (1995).

[20] D. Sudarsky, Class. Quant. Grav. 12, 579 (1995).

[21] J. D. Bekenstein, Phys. Rev. D 51, R6608 (1995).

[22] T. Matos, F. S. Guzmán, and Darío Nuñez, Phys. Rev. D 62, 061301 (2000)

[23] P. J. E. Peebles, astro-ph/0002495; A. Riotto and I. Tkachev, astro-ph/0003388; J. Goodman astro-ph/0003018

[24] T. Damour and G. Esposito-Farèse, Phys. Rev. Lett. 70, 2220 (1993); ibid Phys. Rev. D 54, 1474 (1996); ibid Phys. Rev. D 58, 042001 (1998).

[25] M. Salgado, D. Sudarky and U. Nucamendi, Phys. Rev. D 58, 124003 (1998).

[26] I. Peña and D. Sudarsky, Class. Quant. Grav. 14, 3131 (1997).

[27] D. Zaritzky, R. Smith, C. S. Frenk and S. D. M. White, Astrophys. J. 405, 464 (1993); D. Zaritzky and S. D. M. White, ibid. 435, 599 (1994).

[28] T. Brainerd, R. Blanford and I. Smail, Astrophys. J. 466, 623 (1996).

[29] A. S. Kulessa and D. Lynden-Bell, Mon. Not. R. Astr. Soc. 255, 105 (1992); C. S. Kochanek, Astrophys. J. 457, 228 (1996).

[30] D. Zaritzky, R. Smith, C. S. Frenk and S. D. M. White, preprint astro-ph/9611199.

[31] Those contributions are: that which is associated to the energy density of the scalar field and the other which is associated to the reduction of the negative binding energy due to the reduction of $G$ due to a nonzero value of the scalar field: $G \sim 1 /\left(1+16 \pi \xi \phi^{2}\right)$ with $\xi>0$.

[32] M. Barriola and A. Vilenkin, Phys. Rev. Lett. 63, 341 (1989). 
[33] D. Harari and C. Loustò, Phys. Rev. D 42, 2626 (1990).

[34] U. Nucamendi, M. Salgado, and D. Sudarsky, Phys. Rev. Lett. 84, 3037 (2000).

[35] A. Edery, Phys. Rev. Lett. 83, 3990 (1999).

[36] J. D. Bekenstein, M. Milgrom, and R. H. Sanders, Phys. Rev. Lett. 85, 1346 (2000).

[37] W. A. Hiscock, Phys. Rev. Lett. 64, 344 (1990).

[38] U. Nucamendi and D. Sudarsky, Class. Quant. Grav. 14, 1309 (1997).

[39] R. M. Wald (private communication).

[40] D. P. Bennett and S. Hong Rhie, Phys. Rev. Lett. 65, 1709 (1990).

[41] W. Press, B.P. Flannery, S.A. Teukolsky, W.T. Vetterling, Numerical Recipes, The art of scientific computing, Cambridge University Press, 1986, p. 578.

[42] M. Colpi, S. L. Shapiro, and I. Wasserman, Phys. Rev. Lett. 57, 2485 (1986)

[43] W. L. Freedman et al., Nature 371757 (1994); M. J. Pierce et al., Nature 371385 (1994)

[44] J. Binney and S. Tremaine, Galactic Dynamics, Princeton University Press, Princeton, (1987).

[45] E. Seidel and W.-M. Suen, Phys. Rev. Lett. 72, 2516 (1994)

[46] F. E. Schunk, and E. Mielke, Proc. 8th M. Grossmann Meeting, (ed. T. Piran), World Scientific, p. 1441, Singapore, 1999.

[47] I. Peña and D. Sudarsky, In preparation.

[48] F. E. Schunk, in Proceeding of the 8th Marcel Grossman Meeting (ed. T. Piran), World Scientific, p. 1441, Singapore, 1999; F. E. Schunk, astro-ph/9802258

[49] T. Barreiro, E. J. Copeland, and N. J. Nunes, Phys. Rev. D 61, 127301 (2000)

[50] R. B. Tully and J. R. Fisher, A\&A, 54, 661 (1977).

[51] P. de Berdarnis et al., Nature 404, 955 (2000); S. Hanany et al., Atrophys. J 545, L5 (2000); U.L. Pen, U. Seljak, and N. Turok, Phys. Rev. Lett. 79, 1611 (1997); R. Durrer, M. Kunz, and A. Melchiorri, Phys. Rev. D 59, 123005 (1999).

[52] A. G. Riess et al., The Astronomical Journal 116, 1009 (1998); S. Perlmutter et al., Astrophys. J. 517, 565 (1999) 


\section{FIGURES}

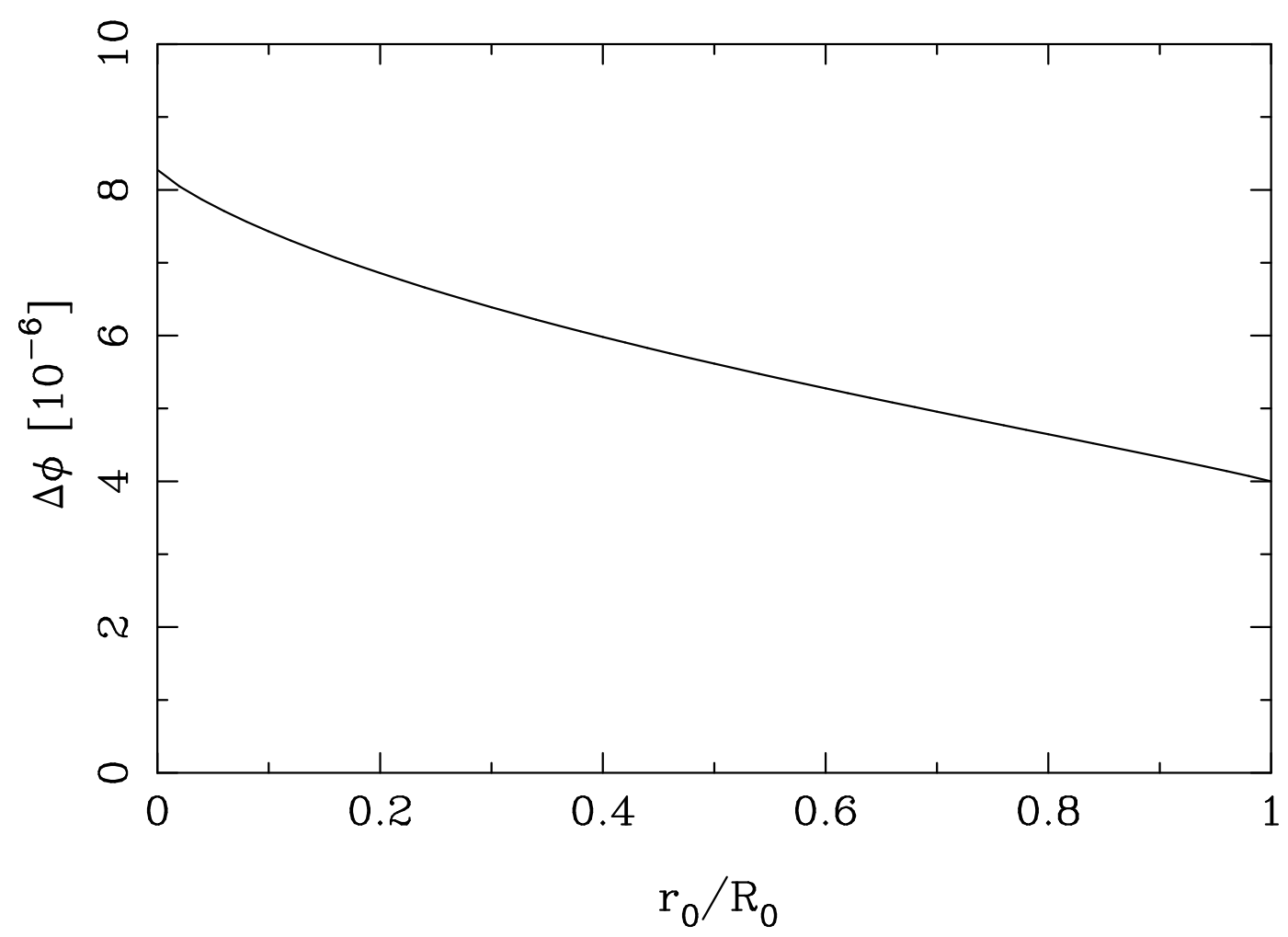

FIG. 1. Bending angle of the light as a function of parameter $r_{0} / R_{0}$. 


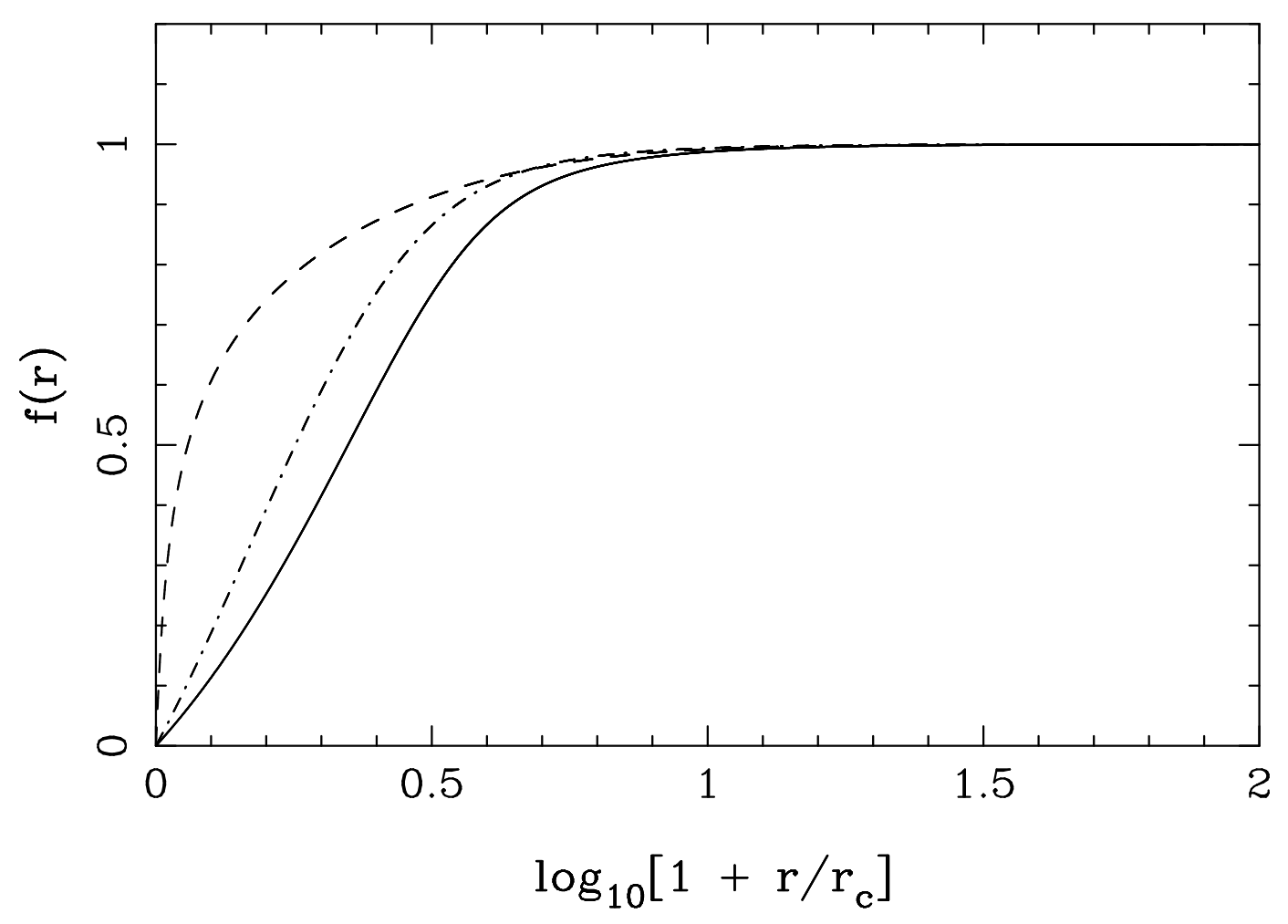

FIG. 2. The figure depicts the global monopole field $f(r)$ for $\xi=0, \alpha=0.795$ (solid line), $\xi=2, \alpha=0.1$ (dashed line), and $\xi=0.3, \alpha=0.79$ (dash-dotted line). Here $r_{c} \equiv\left(\eta \lambda^{1 / 2}\right)^{-1}$. 


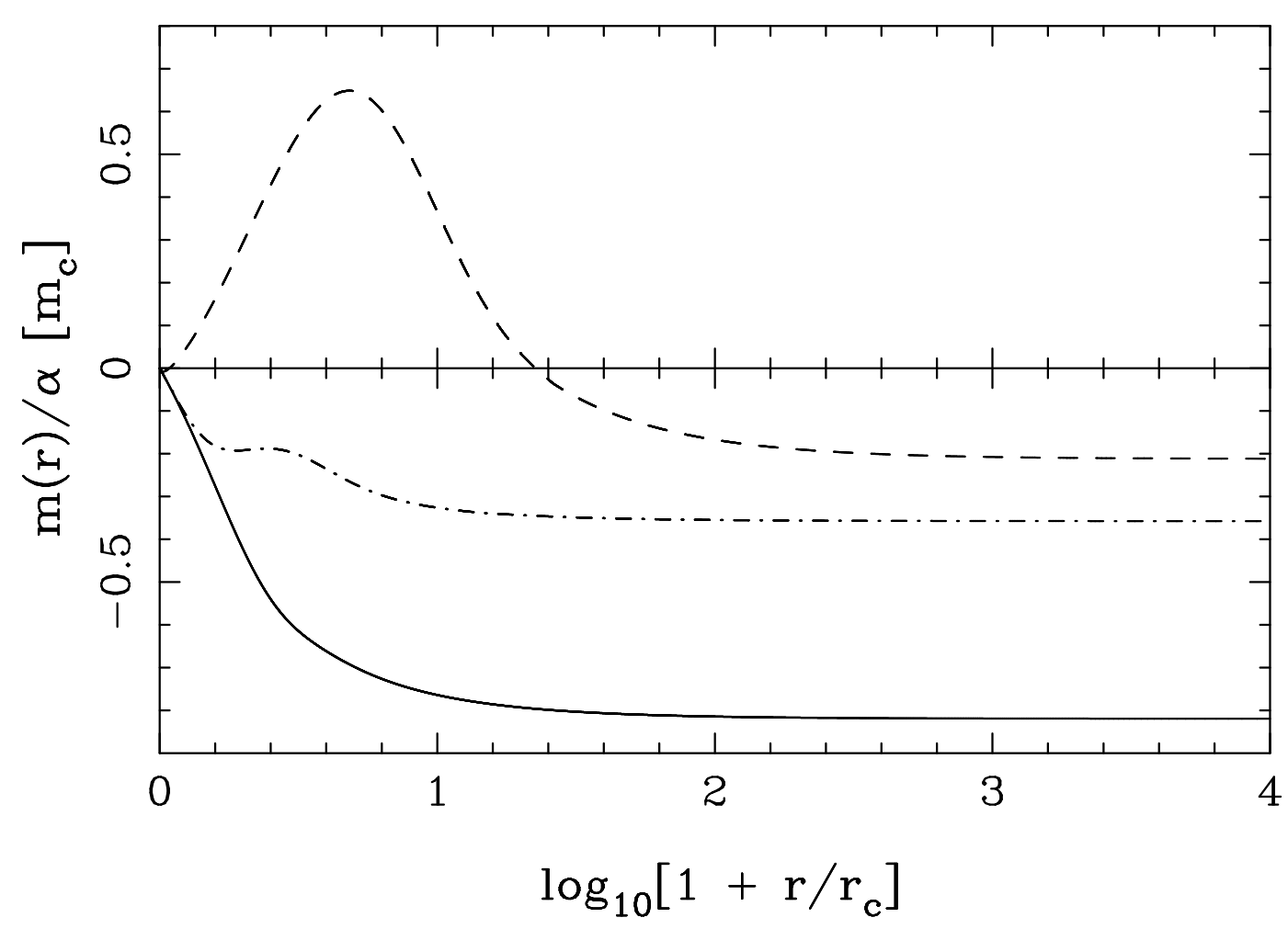

FIG. 3. Mass profile $m(r)$ for $\xi=0, \alpha=0.795$ (solid line), $\xi=2, \alpha=0.1$ (dashed line), and $\xi=0.3, \alpha=0.79$ (dash-dotted line). Asymptotically this quantity provides the ADM mass of the configuration. Here $m_{c} \equiv\left(G_{0} \eta \lambda^{1 / 2}\right)^{-1}$.
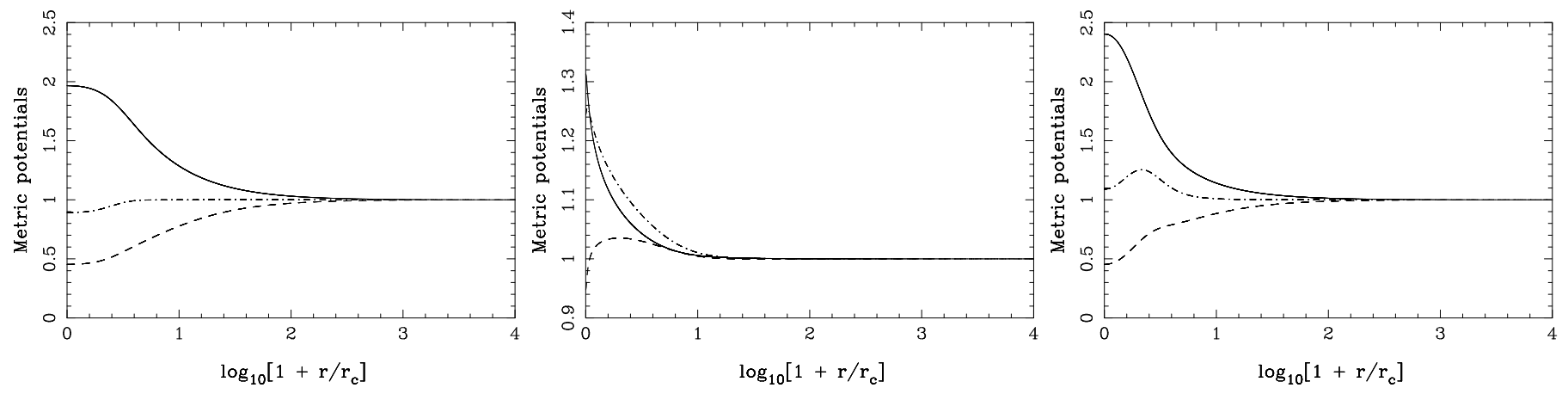

FIG. 4. Metric potentials $N(r) /(1-\alpha)^{1 / 2}$ (solid lines), $A(r)(1-\alpha)^{1 / 2}$ (dashed lines), and the product $A N$ (dash-dotted lines) for three different configurations. The left panel corresponds to $\xi=0, \alpha=0.795$, the middle panel to $\xi=2, \alpha=0.1$, and the right panel to $\xi=0.3, \alpha=0.79$. Note that $A N$ aproaches to unit "outside" the monopole core, while the metric potentials $N$ and $A$ tend to the asimptotically-flat-but-for-a-deficit-angle values far from the origin. 


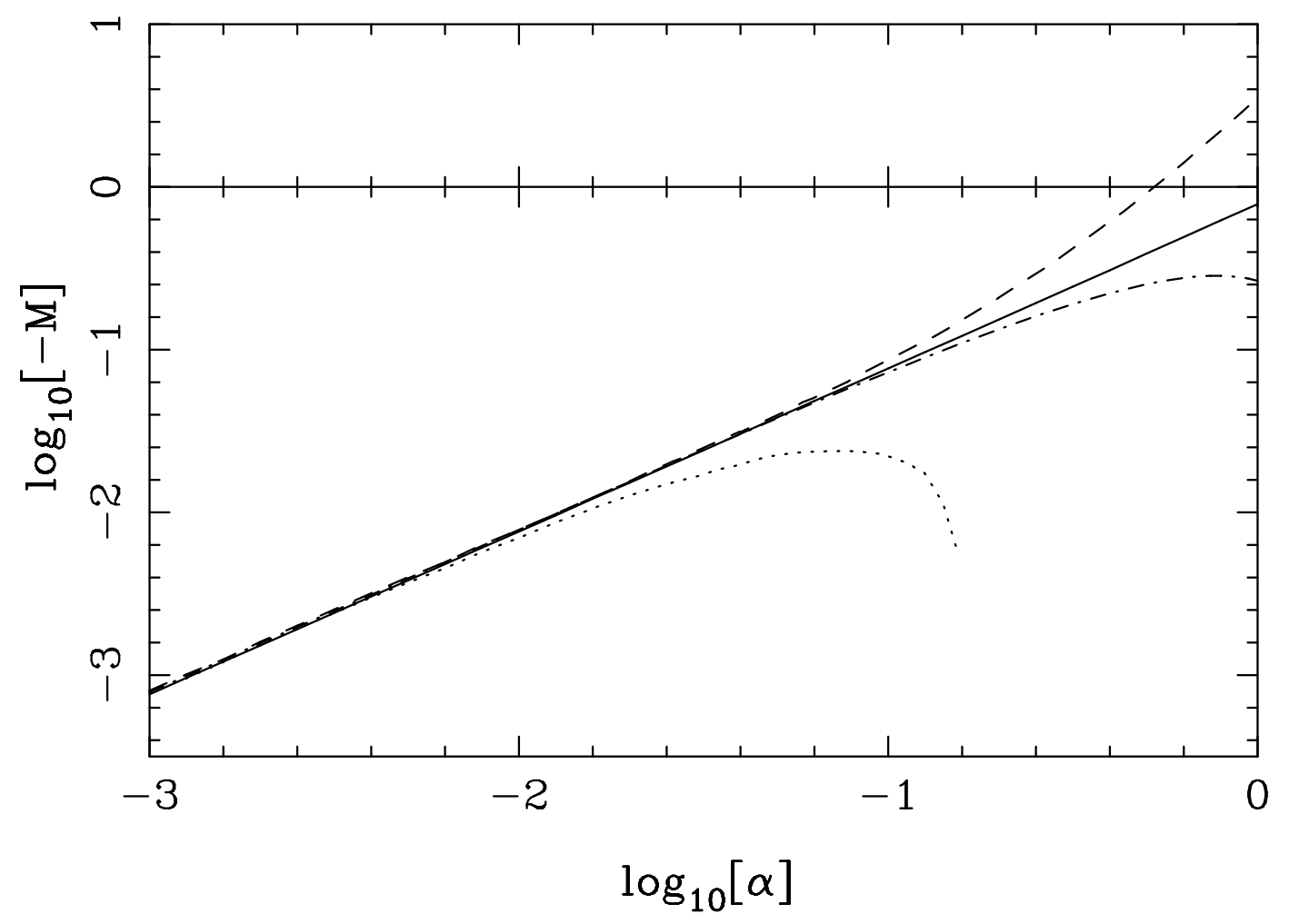

FIG. 5. ADM mass of configurations for different values of the deficit angle for $\xi=0$ (solid line), $\xi=-2$ (dashed line), $\xi=0.3$ (dash-dotted line) and $\xi=2$ (dotted line). 


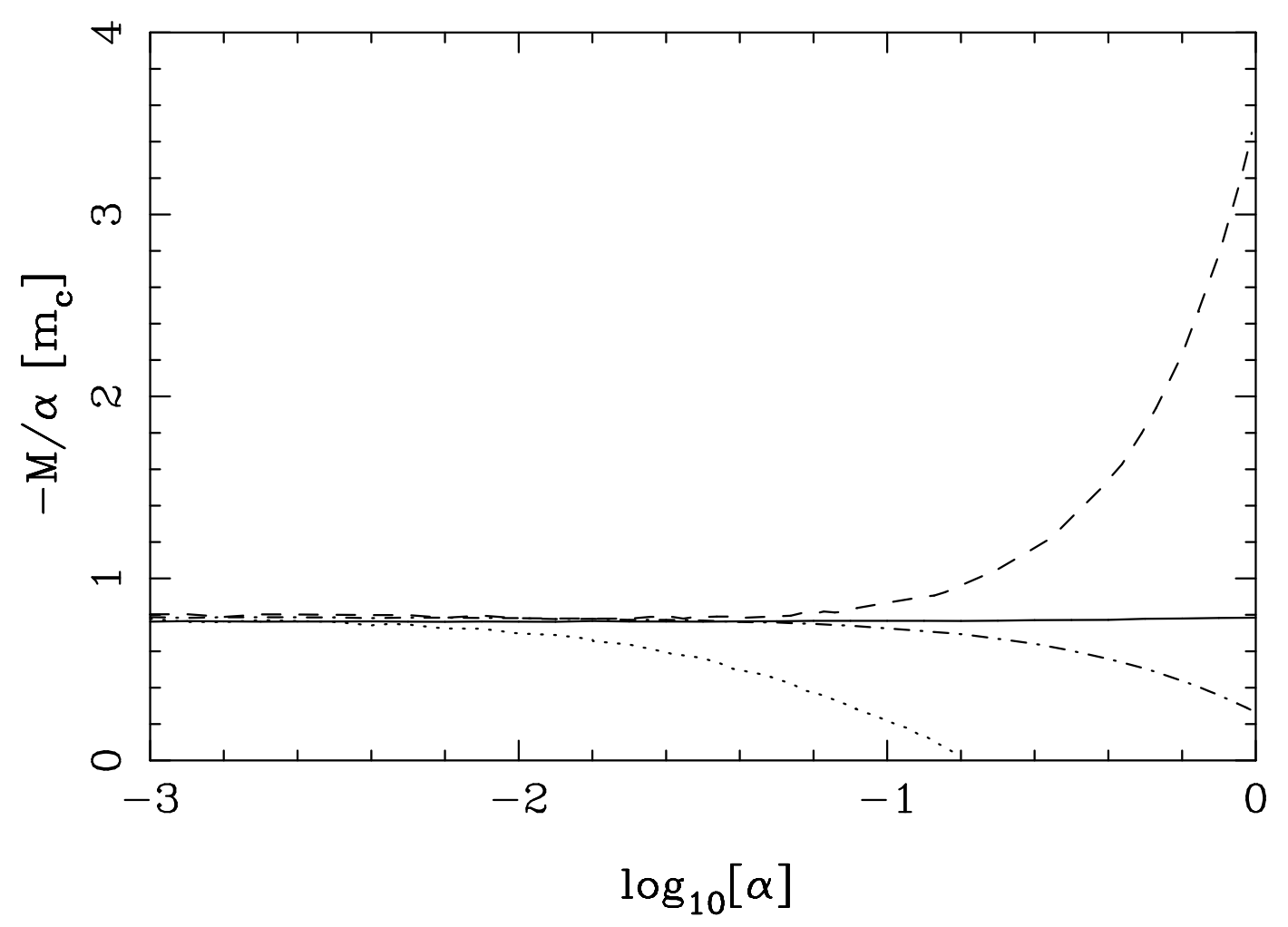

FIG. 6. ADM mass-deficit angle rate of configurations for different values of the deficit angle for $\xi=0$ (solid line), $\xi=-2$ (dashed line), $\xi=0.3$ (dash-dotted line), and $\xi=2$ (dotted line). 


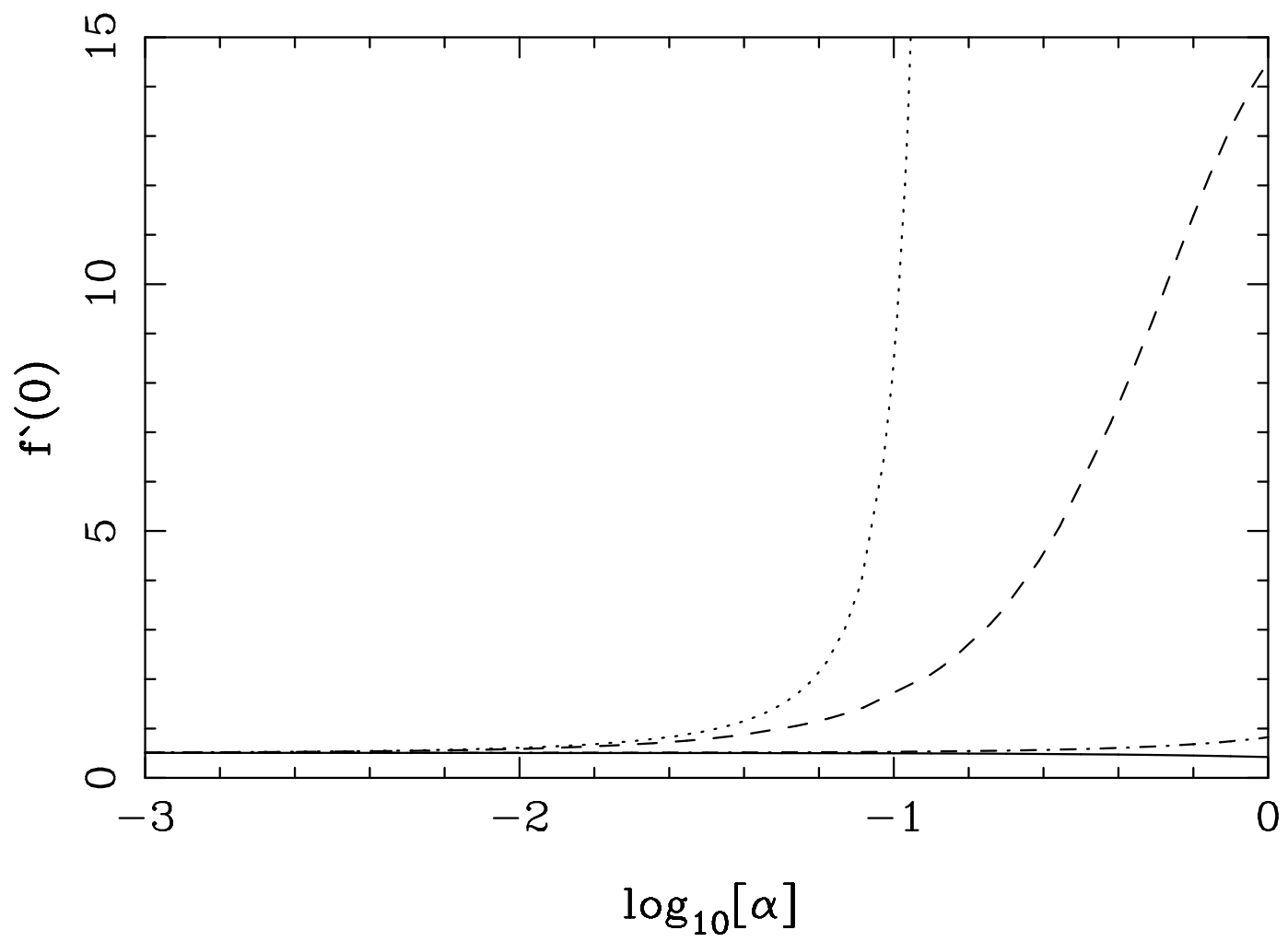

FIG. 7. Derivative of the global monopole at $r=0$ for different values of the deficit angle for $\xi=0$ (solid line), $\xi=-2$ (dashed line), $\xi=0.3$ (dash-dotted line), and $\xi=2$ (dotted line).
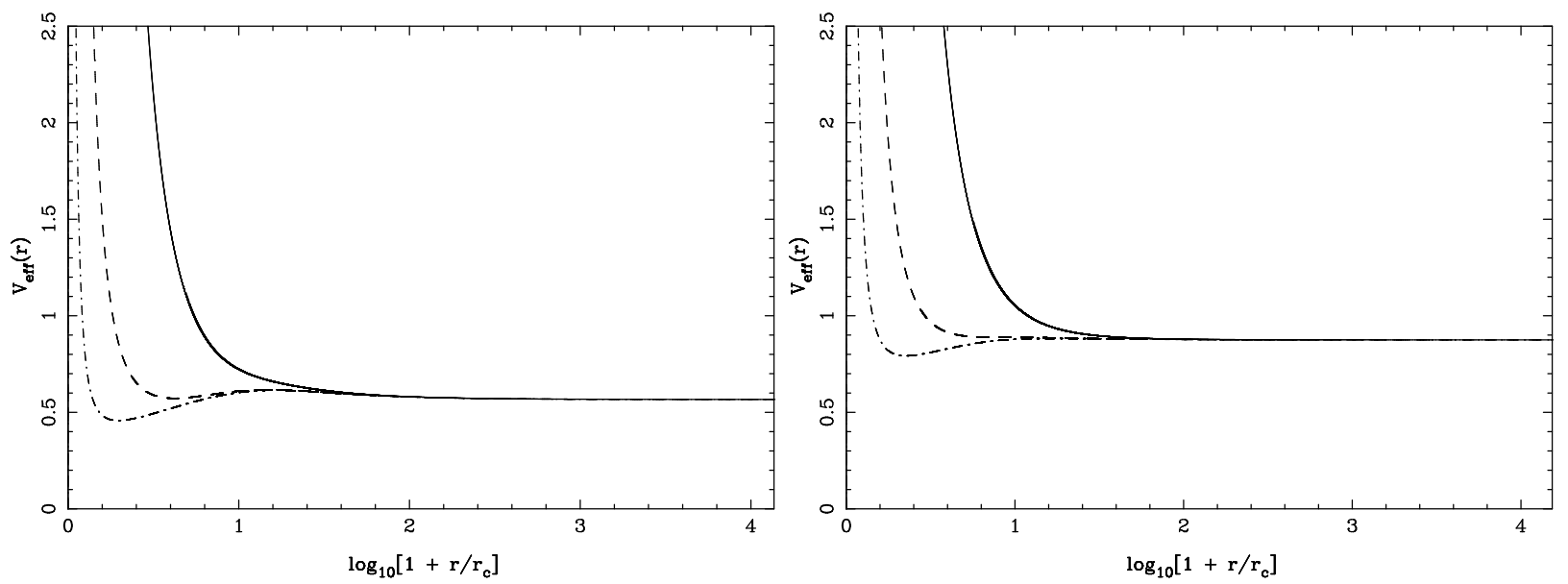

FIG. 8. Functional dependence of the effective potential $V_{\text {eff }}$ vs $\tilde{r}$ for the case $\xi=-2$, $\alpha=0.43$ (left panel) and $\alpha=0.125$ (right panel). For each configuration we show three values of the angular momentum: $L=4$ (solid line), $L=1$ (dashed line) and $L=0.3$ (dash-dotted line). Here $r_{c} \equiv\left(\eta \lambda^{1 / 2}\right)^{-1}$. 


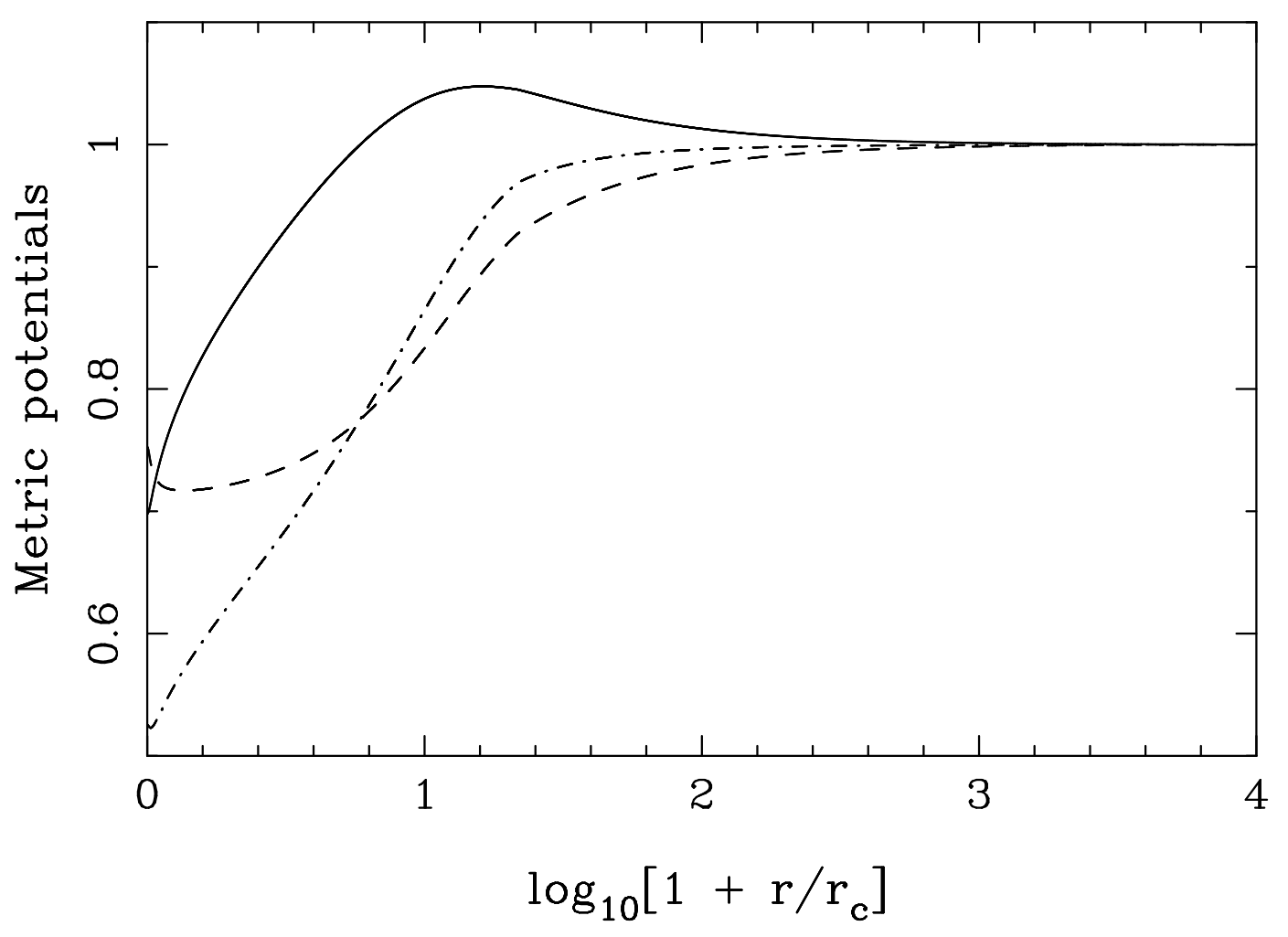

FIG. 9. Same as fig, 4 for $\xi=-2, \alpha=0.43$.
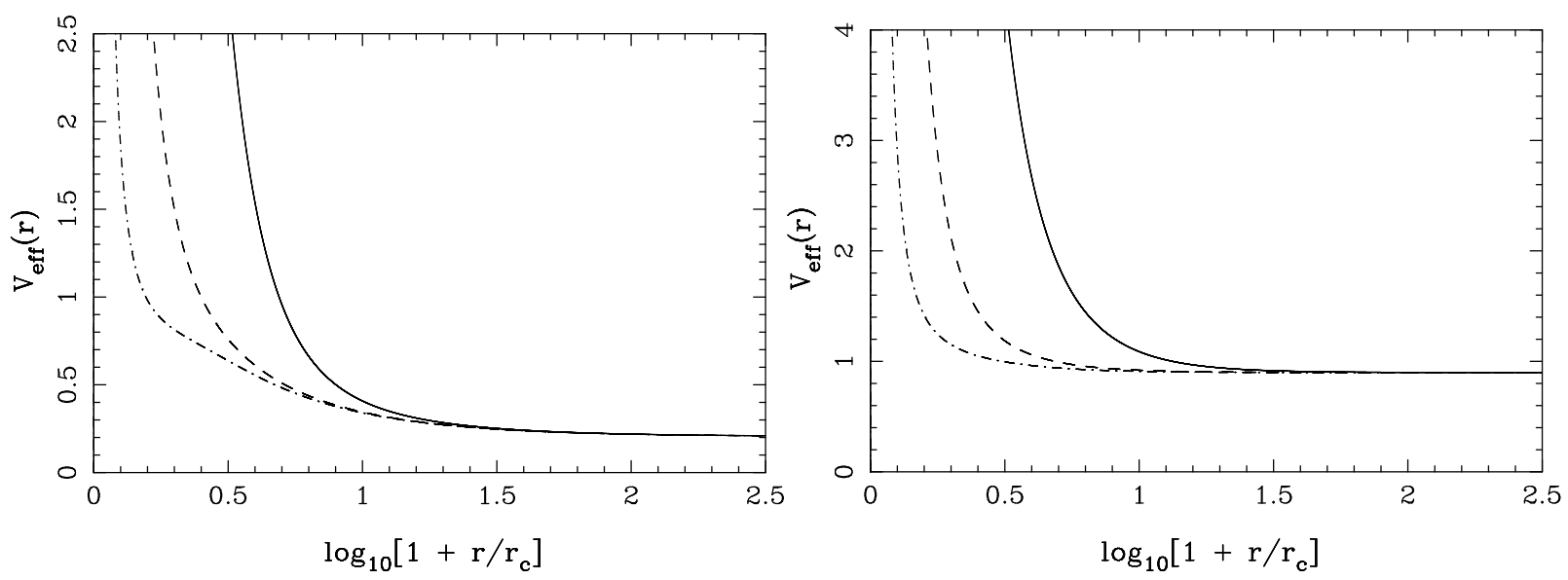

FIG. 10. Same as fig. 8 for $\xi=0, \alpha=0.795$ (left panel) and for $\xi=2, \alpha=0.1$ (right panel). 

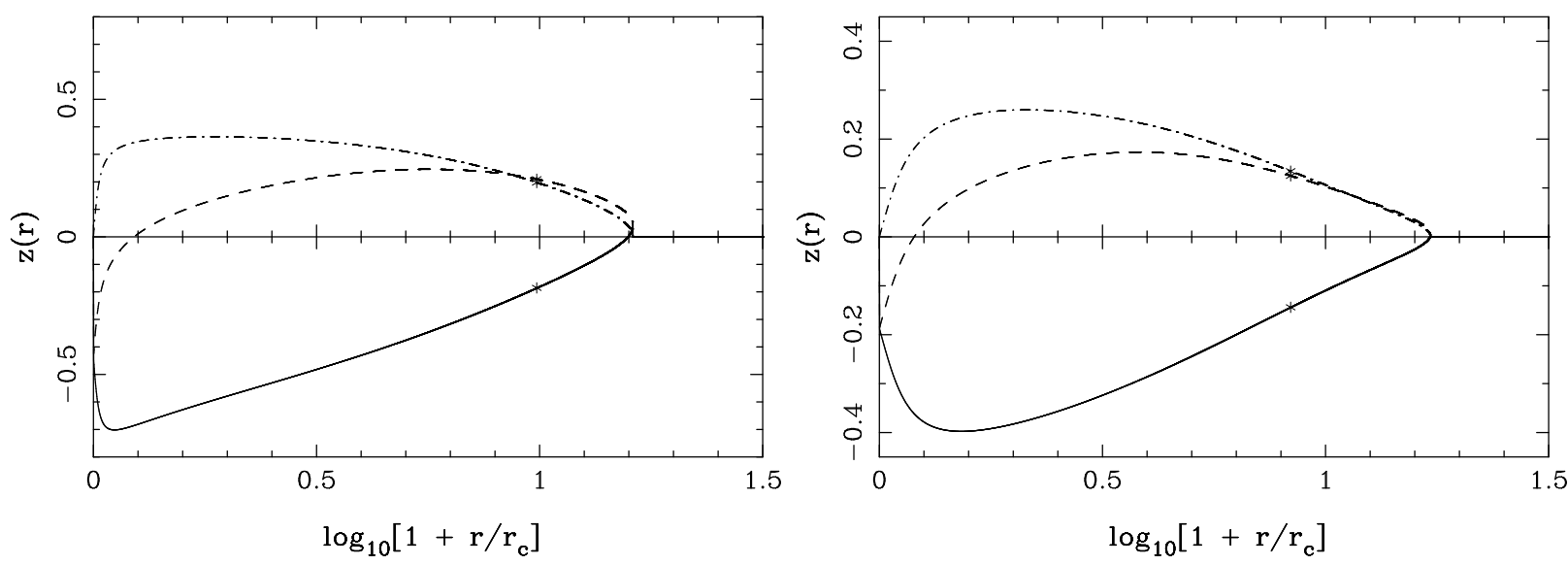

FIG. 11. Functional dependence of $z_{+}$(dashed line), $z_{-}$(solid line) and $z_{D}$ (dash-dotted line) vs $\tilde{r}$ for circular orbits in the case $\xi=-2, \alpha=0.43$ (left panel) and $\alpha=0.125$ (right panel). The asterisk depicts the location of the radius beyond which the stable circular orbits cease to exist $\left(r \sim 9 r_{c}\right)$.

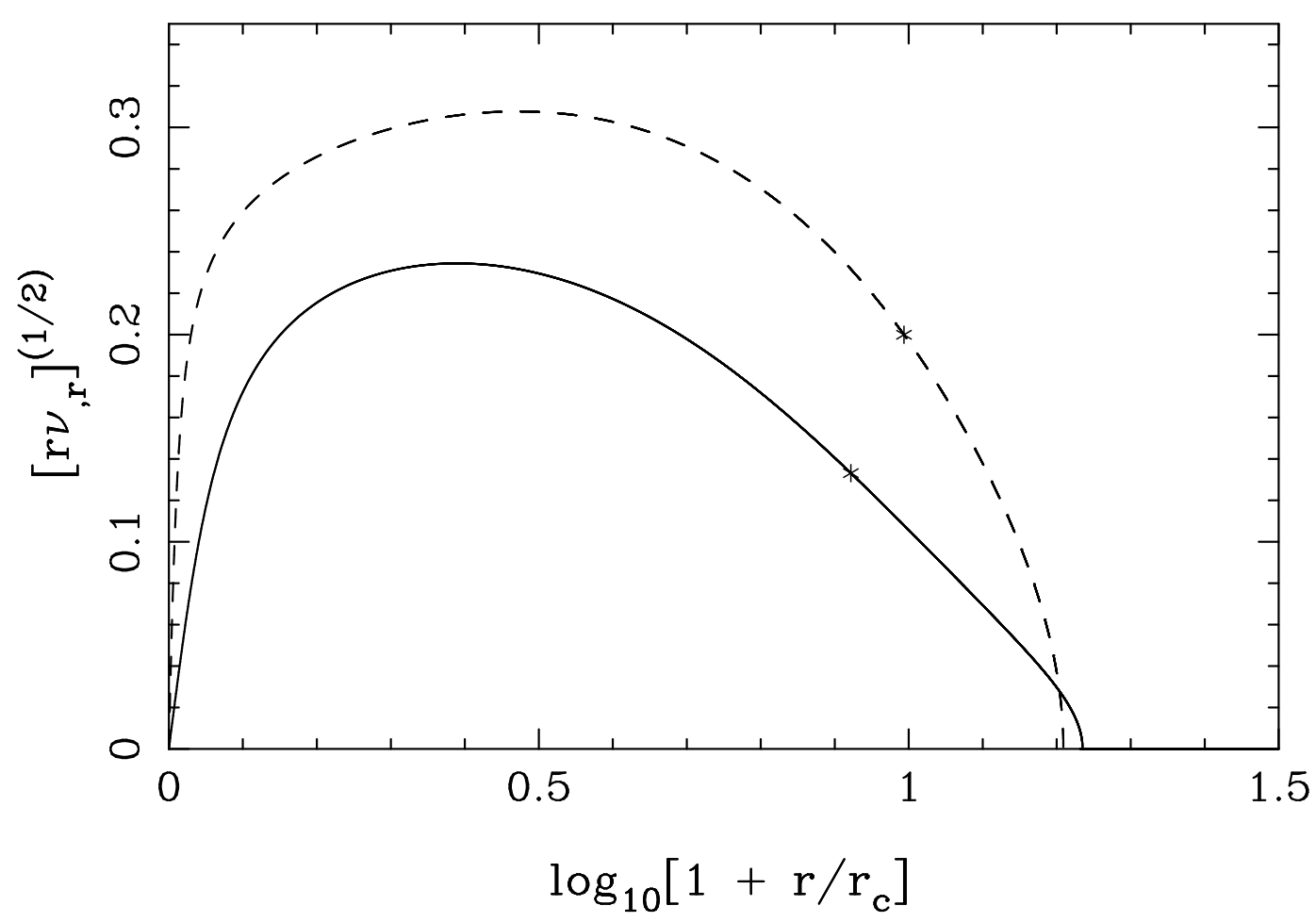

FIG. 12. Tangential velocity $v=\left(r \partial_{r} N / N\right)^{1 / 2}$ in units of $c$ for $\xi=-2, \alpha=0.125$ (solid line) and $\alpha=0.43$ (dashed line). The asterisk depicts the location of the radius beyond which the stable circular orbits cease to exist. 